\title{
A Parametric Method for Ranking Intuitionistic Fuzzy Numbers and Its Application to Solve Intuitionistic Fuzzy Network Data Envelopment Analysis Models
}

\author{
B. Shakouri, ${ }^{1}$ R. Abbasi Shureshjani $\mathbb{D D}^{2}{ }^{2}$ B. Daneshian, ${ }^{1,3}$ and F. Hosseinzadeh Lotfi ${ }^{4}$ \\ ${ }^{1}$ Department of Mathematics, Tabriz Branch, Islamic Azad University, Tabriz, Iran \\ ${ }^{2}$ Department of Management, Humanities College, Hazrat-e Masoumeh University, Qom, Iran \\ ${ }^{3}$ Department of Mathematics, Central Tehran Branch, Islamic Azad University, Tehran, Iran \\ ${ }^{4}$ Department of Mathematics, Science and Research Branch, Islamic Azad University, Tehran, Iran \\ Correspondence should be addressed to R. Abbasi Shureshjani; roohollah31@gmail.com
}

Received 17 April 2020; Revised 2 August 2020; Accepted 15 August 2020; Published 2 September 2020

Academic Editor: Qingling Wang

Copyright (c) 2020 B. Shakouri et al. This is an open access article distributed under the Creative Commons Attribution License, which permits unrestricted use, distribution, and reproduction in any medium, provided the original work is properly cited.

In this study, a new parametric method is proposed to rank intuitionistic fuzzy numbers in a general form. One of the advantages of the proposed method is that the decision maker's idea is taken into account by selecting appropriate amounts of decision level and hesitation degree parameters. In some illustrative examples, the superiority of the proposed method over some other approaches is demonstrated. Furthermore, to show the ability of the method to solve intuitionistic fuzzy optimization problems, the proposed method is applied to solve intuitionistic fuzzy network data envelopment analysis (IFNDEA) problems. Also, in three appropriate examples, the validity of the suggested method and its capacity to solve real-world problems are illustrated.

\section{Introduction}

Intuitionistic fuzzy sets (IFSs), first proposed by Atanassov $[1,2]$, are a generalization of Zadeh's fuzzy sets $[3,4]$ to model noncrisp and uncertain sets. For these sets, we define both membership and nonmembership functions. In this case, we will be able to define a hesitation function that is the difference between the "membership function" and "one minus nonmembership function." Thus, by IFSs, we can efficiently model imperfect information. Many researchers provided enormous works on IFSs in both theory and applications (see e.g., [5-8]). As an application, Akram et al. [9] proposed a novel decision-making method based on hypergraphs in the intuitionistic fuzzy environment and applied it to real-life problems.

In 1994, by proposing intuitionistic fuzzy numbers (IFNs), Burillo et al. [10] generalized a fuzzy numbers concept and paved the way for applying intuitionistic fuzzy logic in real-life problems such as decision-making and risk analysis. For example, Liu and Wang [11] and Wang and Liu
[12] proposed new Schweizer-Sklar Maclaurin and Einstein operation rules for IFNs and applied them to solve real-life decision-making problems.

Ranking IFNs is one of the important subjects in IFS theory, and a considerable number of articles have been published in this field. In these papers, different approaches are employed to compare and rank IFNs. For example, Grzegorzewski [13] generalized the ranking approach of fuzzy numbers in Grzegorzewski [14] and suggested a new method for ranking IFNs by defining concepts of expected interval and expected value. Later, Ye [15] calculated the expected value of a TraIFN that was introduced by Grzegorzewski [13] and applied it to a trapezoidal intuitionistic fuzzy multicriteria decision-making problem. Using four pairwise ranking functions, Mitchel [16] defined the average intuitionistic fuzzy rank index and proposed an approach to compare each pair of IFNs. Using score and accuracy functions of trapezoidal intuitionistic fuzzy numbers (TraIFNs), Jianqiang and Zhong [17] proposed a stepwise ranking method and applied it to a multicriteria 
decision-making problem. Wang and Zhang [18] ranked TraIFNs by converting them into interval numbers. Nehi [19] proposed a generalization of the characteristic value first suggested by Chiao [20] and ranked IFNs. In the work of Nan et al. [21], by introducing average indexes of membership and nonmembership functions, TraIFNs are ranked, and then, the approach is applied to matrix games with payoffs. Later, in a note, Verma and Kumar [22] modified some mathematical incorrect assumptions in the work of Nan et al. [21]. Value and ambiguity concepts were first introduced by Delgado et al. [23], and employing these concepts is a famous approach for ranking IFNs (see e.g., [24-28]). For example, in the work of $\mathrm{Li}$ [26], value and ambiguity are used to rank nonnormal triangular intuitionistic fuzzy numbers (TriIFNs), and then, the proposed method is applied to a personnel selection problem. In addition, Chutia and Saikia [25] generalized value and ambiguity concepts for membership and nonmembership functions and proposed a ranking method for IFNs using $\alpha$ and $\beta$ cuts. They also applied the proposed method in a risk analysis problem with intuitionistic fuzzy data. Wan [29], using possibility mean, possibility variance, standard deviation, and variance coefficient of TrilFNs, introduced possibility variance coefficients of membership and nonmembership functions to create a lexicographic ranking method. Das and Guha [30] ranked TraIFNs by calculating the centroid points and compared them. Li and Chen [31] proposed a distance index between two arbitrary TraIFNs based on $\alpha$-cuts, which was, then, applied to the intuitionistic fuzzy multicriteria group decision-making problem. In the work of Lakshmana Gomathi Nayagam et al. [32], eight score functions are defined and applied to rank TraIFNs. By appropriate examples, the method is compared with some other approaches in this field. Also, Nayagam et al. [33] generalized a lexicographic ranking method to propose a total ordering of IFNs using $\alpha$-cut and $\beta$-cut sets. In another work, Nayagam et al. [28] proposed a parametric score function using improved value and ambiguity indexes to rank TraIFNs and applied it to solve a multicriteria decision-making problem. Aggarwal and Gupta [34] defined an index to rank generalized symmetrical TraIFNs and solved an intuitionistic fuzzy solid transportation problem. Prakash et al. [35] introduced a ranking method by calculating the centroid of TraIFNs. Singh and Yadav [36] defined score and accuracy indices for the LR type of normal IFNs and proposed an order relation. Furthermore, by applying these indices, they proposed a method for solving a fully intuitionistic fuzzy linear-programming problem. Later, Canedo and Morales [37] analyzed this approach and modified it to obtain unique optimal solutions. Darehmiraki [38] extended the method by Shureshjani and Daremiraki [39] to rank IFNs and applied it to the partner selection problem. Table 1 summarizes the abovementioned studies based on the relevant themes. Although many methods have been proposed, there is still not a method to rank different kinds of IFNs, which can also be easily employed in optimization problems such as Data Envelopment Analysis (DEA) under intuitionistic fuzzy environment via the decision-maker idea.
DEA is a nonparametric and linear-programming-based approach for evaluating the efficiency of decision-making units (DMUs) with multiple inputs and outputs data. From the first work by Charnes et al. [48], there are many studies on both theory and applications in this field. In DEA models, a DMU is considered as a black box with its interior structures not taken into account. However, in many applications, DMUs have important interior structures, and ignoring them has led to inappropriate results. Network DEA is an attempt to consider the interior structures of DMUs. Among all the proposed structures, the simplest and most basic network DEA model is two-stage network DEA models. Also, in conventional DEA models, all the data should be crisp and certain which is not always possible in real applications. To solve these problems, fuzzy numbers and IFNs, fuzzy DEA, and intuitionistic fuzzy DEA (IFDEA) models are proposed, respectively (see Emrouznejad et al. [49] for a literature review on fuzzy DEA models and its applications until 2013). Fuzzy DEA can efficiently model DEA problems with noncrisp data, but when we face both noncrisp and uncertain data, IFDEA models are the best alternative. Although many papers have been published on fuzzy DEA, the proposed works in the IFDEA field are limited. For example, Xu et al. [40] introduced a fuzzy superefficient cross-DEA model to measure the efficiency of logistics enterprises using an interval-valued intuitionistic fuzzy Bayesian network. Using a weighted aggregation operator, Razavi Hajiagha et al. [41] proposed an approach to evaluate the efficiency of DMUs in an intuitionistic fuzzy BCC model. Puri and Yadav [42], by applying the expected interval and expected value (Grzegorzewski [13]), proposed an index to estimate triangular intuitionistic fuzzy input and output data in an IFDEA model. Similar to the work of Puri and Yadav [42], by applying the expected value (Grzegorzewski [13]), Singh [43] transformed an intuitionistic fuzzy DEA/AR model into a conventional DEA/AR model. Using $\alpha$-cut and $\beta$-cut concepts in IFS theory, Arya and Yadav [44] introduced lower and upper bounds for the efficiency of DMUs in intuitionistic fuzzy SBM and superefficiency intuitionistic fuzzy SBM models. In another paper, Arya and Yadav [45] proposed appropriate models based on $\alpha$-cuts and $\beta$-cuts to provide lower and upper bounds of efficiency measures in IFDEA, and then, by applying the index proposed by Chen and Klein [50], they ranked DMUs. Also, their method was applied to evaluate and rank 16 hospitals in India. Moreover, Arya and Yadav [46] developed intuitionistic fuzzy BCC and intuitionistic fuzzy superefficiency BCC models with triangular IF inputs and outputs data. They developed the proposed approach to determine the efficiencies and rankings of DMUs in the presence of infeasibility. Finally, in Ameri et al. [47], by substituting the IF inputs and IF outputs of a parallel IFNDEA model with their assigned expected values (Grzegorzewski [13]), the parallel IFNDEA model transforms into a linear program. In a case study, their method was applied for the self-assessment of an Iranian hospital (See Table 1).

Two important limitations of the abovementioned approaches in IFDEA are that, almost all of them work for a 
TABLE 1: Summary of the literature review.

Ranking IFNs

Studies to develop a ranking method using the expected value concept

Studies to develop a ranking method using the statistical viewpoint Studies to develop a ranking method using average indexes of membership and nonmembership functions

Studies to develop a ranking method using the distance index

Studies to develop a ranking method using score and accuracy indices

Studies to develop a ranking method using value and ambiguity concepts

Studies to develop a ranking method using a centroid concept Studies to develop a ranking method using an integral value of membership and nonmembership functions

\section{IFDEA model}

A superefficient cross-DEA model based on the Bayesian network in the interval-intuitionistic fuzzy environment

An intuitionistic fuzzy BCC model

Optimistic and pessimistic IFDEA models with triangular

intuitionistic fuzzy data

An intuitionistic fuzzy DEA/AR model with triangular intuitionistic fuzzy data

Intuitionistic fuzzy SBM and superefficiency intuitionistic fuzzy SBM models with triangular intuitionistic fuzzy data

An intuitionistic fuzzy CCR model with triangular intuitionistic

fuzzy data

Intuitionistic fuzzy BCC and intuitionistic fuzzy superefficient BCC models with triangular intuitionistic fuzzy data

IFNDEA models

Study to develop a parallel intuitionistic fuzzy network DEA model
Grzegorzewski [13], Ye [15], and Jianqiang and Zong [17]

Mitchell [16] and Wan [29]

Nan et al. [21] and Verma and Kumar [22]

Wang and Zhang [18], Aggarwal and Gupta [34], and Li and Chen [31]

Lakshmana Gomathi Nayagam et al. [32], Nayagam et al. [33], Singh and Yadav [36], and Canedo and Morales [37]

Li [26], Li et al. [27], Chutia and Chutia [24], Nayagam et al. [28], and Chutia and Saikia [25]

Das and Guha [30] and Prakash et al. [35]

Nehi [19] and Darehmiraki [38] specific group of IFNs (triangular IFNs) only and the decision maker does not play any role in the decision-making process. In addition, they are not easily generalizable to intuitionistic fuzzy network DEA (IFNDEA) models. Despite a large number of papers published in the IFDEA field, there has been one published paper (by Ameri et al. [47]) in the IFNDEA field.

The main motive of this study is to overcome the shortcomings listed above by assigning an appropriate parametric index to IFNs considering two main concepts in IFSs, i.e., alpha cut and hesitation degree. In this case, by proper selection of the decision level (alpha cut) and the hesitation degree, the decision maker's idea is accounted for in the decision-making process, and less information is lost in IFNs. This parametric index can be applied to compare and rank IFNs.

Also, by substituting the IF data with their assigned parametric indexes, an intuitionistic fuzzy decision-making problem transforms into a parametric decision-making problem that can be easily solved by proper selection of the parameters. In this study, a new parametric method is developed to rank IFNs. Also, some reasonable properties to rank IFNs are examined based on the work of Wang and Kerre [51]. To show the ability of the method in real-life problems, it is applied to solve the IFDEA problem in singlestage, series, and parallel structures. As will be seen, the proposed method is also easily generalizable to other kinds of IFNDEA problems.
The remainder of this research is organized as follows. In Section 2, some preliminaries of IFSs, IFNs, and the related arithmetic operators are introduced. In Section 3, using decision level and hesitation degree concepts, a new parametric method is proposed to rank IFNs in a general form, by appropriate examples, the advantages of the proposed method are illustrated, and the obtained results are finally compared with some other approaches in this field. In Section 4, we applied our proposed method to solve the IFNDEA problem. In this method, IFDEA and IFNDEA models are transformed into parametric DEA and parametric NDEA models, respectively. Three examples illustrate the method. Finally, Section 5 concludes the paper.

\section{Preliminaries}

This section includes some basic concepts and notions. To easily understand the equations in this paper, some mathematical symbols are summarized in Table 2 .

Definition 1 (see $[1,2]$ ). Let $X$ be a fixed universe. An IFS $\widetilde{A}$ in $X$ is given as

$$
\left\{\left\langle x, \mu_{\widetilde{A}}(x), v_{\widetilde{A}}(x)\right\rangle \mid x \in X\right\}
$$


TABLE 2: Mathematical symbols utilized in the following formulas.

\begin{tabular}{|c|c|}
\hline Mathematical symbols & Meanings \\
\hline$\widetilde{A}$ & An intuitionistic fuzzy number \\
\hline$\mu_{\widetilde{A}}(x)$ & Membership function of $\widetilde{A}$ \\
\hline$v_{\widetilde{A}}(x)$ & Nonmembership function of $\tilde{A}$ \\
\hline$\pi_{\widetilde{A}}^{A}(x)$ & Hesitation function of $\widetilde{A}$ \\
\hline$w^{A}$ & Maximum degree of the membership function \\
\hline$u$ & Minimum degree of the nonmembership function \\
\hline$\tilde{A}_{\alpha}$ & $\alpha$-cut set of $\widetilde{A}$ \\
\hline$\tilde{A}_{\beta}^{\alpha}$ & $\beta$-cut set of $\tilde{A}$ \\
\hline$\tilde{A}_{\alpha, \beta}^{\beta}$ & $(\alpha, \beta)$-cut set of $\tilde{A}$ \\
\hline$S_{\alpha, k}(\widetilde{A})$ & The assigned function to $\widetilde{A}$ for the selected decision level $(\alpha)$ and hesitation degree $(k)$ \\
\hline $\bar{m}(r), n(r)$, and $\overline{n^{\prime}}(r)$ & Bounded monotonic decreasing left continuous functions \\
\hline$\underset{\sim}{m}(r), \overline{\bar{n}}(r)$, and $\underline{n}^{\prime}(r)$ & Bounded monotonic increasing left continuous functions \\
\hline$\tilde{\tilde{\theta}}_{o}$ & Intuitionistic fuzzy efficiency score of the decision-making unit under evaluation \\
\hline$\tilde{x}_{i j}$ & The $i^{\text {th }}$ intuitionistic fuzzy input data of the $j^{\text {th }}$ decision-making unit \\
\hline$\tilde{y}_{r j}$ & The $r^{\text {th }}$ intuitionistic fuzzy output data of the $j^{\text {th }}$ decision-making unit \\
\hline$\widetilde{z}_{d j}$ & The $d^{\text {th }}$ intuitionistic fuzzy intermediate data of the $j^{\text {th }}$ decision-making unit \\
\hline$v_{i}$ & The given weight to intuitionistic fuzzy input data $i$ \\
\hline$u_{r}$ & The given weight to intuitionistic fuzzy output data $r$ \\
\hline$w_{d}$ & The given weight to intuitionistic fuzzy intermediate data $d$ \\
\hline
\end{tabular}

where $\mu_{\widetilde{A}}(x), v_{\widetilde{A}}(x)$ are the degrees of membership and nonmembership functions from $X$ to $[0,1]$, respectively. Also, $0 \leq \mu_{\widetilde{A}}(x), v_{\widetilde{A}}(x) \leq 1$ and $0 \leq \mu_{\widetilde{A}}(x)+v_{\widetilde{A}}(x) \leq 1$.

Definition 2 (see $[1,2]$ ). The hesitation function of an IFS $\widetilde{A}$ is defined as

$$
\pi_{\widetilde{A}}(x)=1-\mu_{\widetilde{A}}(x)-v_{\widetilde{A}}(x) .
$$

It is clear that $0 \leq \pi_{\widetilde{A}}(x) \leq 1$. This function indicates the hesitation degree of an element $x$ (indeterminacy) belonging or not belonging to $\widetilde{A}$.

In fuzzy sets, we do not have a hesitation degree (hesitation degree is zero). Thus, we can consider each fuzzy set as a special case of an IFS as

$$
\left\{\left\langle x, \mu_{\widetilde{A}}(x), 1-\mu_{\widetilde{A}}(x)\right\rangle \mid x \in X\right\} .
$$

Definition 3 (see [5]). An IFN $\widetilde{A}=\left(\mu_{\widetilde{A}}, v_{\widetilde{A}}\right)$ is an IFS in the set of real numbers $\mathbb{R}$ with the membership function as

$$
\mu_{\widetilde{A}}(x)= \begin{cases}f_{\widetilde{A}}(x), & a \leq x \leq b, \\ w, & b \leq x \leq c, \\ g_{\widetilde{A}}(x), & c \leq x \leq d, \\ 0, & \text { otherwise }\end{cases}
$$

and the nonmembership function as

$$
v_{\widetilde{A}}(x)= \begin{cases}h_{\widetilde{A}}(x), & a^{\prime} \leq x \leq b^{\prime}, \\ u, & b^{\prime} \leq x \leq c^{\prime}, \\ r_{\widetilde{A}}(x), & c^{\prime} \leq x \leq d^{\prime}, \\ 1, & \text { otherwise, }\end{cases}
$$

where $f_{\widetilde{A}}$ and $r_{\widetilde{A}}$ are nondecreasing continuous functions from $\mathbb{R}$ to $[0,1]$ and $g_{\widetilde{A}}$ and $h_{\widetilde{A}}$ are nonincreasing continuous functions from $\mathbb{R}$ to $[0,1]$. Also, $a^{\prime} \leq a, b^{\prime} \leq$ $b \leq c \leq c^{\prime}, d \leq d^{\prime}$. In this definition, $w$ is the maximum degree of the membership function and $u$ is the minimum degree of the nonmembership function. So, according to the Definition $1,0 \leq w, u \leq 1$ and $0 \leq w+u \leq 1$ (Figure 1).

In Definition 3, if $w=1$ and $u=0$, then, we have a normal IFN.

Considering the abovementioned definition (Definition 3), $\alpha$-cut, $\beta$-cut, and $(\alpha, \beta)$-cut sets of an IFN are defined as follows.

Definition 4 (see [2]). $\alpha$-cut, $\beta$-cut, and $(\alpha, \beta)$-cut sets of an IFN $\widetilde{A}=\left(\mu_{\widetilde{A}}, v_{\widetilde{A}}\right)$ are crisp subsets of $\mathbb{R}$ that are defined as follows:

$\alpha$-cut set: $\widetilde{A}_{\alpha}=\left\{x \mid \mu_{\widetilde{A}}(x) \geq \alpha\right\}, \quad 0 \leq \alpha \leq w, \quad \beta$-cut set: $\widetilde{A}_{\beta}=\left\{x \mid v_{\widetilde{A}}(x) \leq \beta\right\}, u \leq \beta \leq 1$, and $(\alpha, \beta)$-cut set:

$$
\begin{aligned}
& \widetilde{A}_{\alpha, \beta}=\left\{x \mid \mu_{\widetilde{A}}(x) \geq \alpha, \nu_{\widetilde{A}}(x) \leq \beta\right\}, \\
& 0 \leq \alpha \leq w, u \leq \beta \leq 1,0 \leq \alpha+\beta \leq 1 .
\end{aligned}
$$

Li et al. [27] proposed a new approach for defining a TriIFN, and here, we generalized their approach for TraIFNs as follows:

Definition 5. A TraIFN $\widetilde{A}$ is an IFN with membership and nonmembership functions as follows:

$$
\mu_{\widetilde{A}}(x)= \begin{cases}\frac{w(x-a)}{b-a}, & a \leq x \leq b, \\ w, & b \leq x \leq c, \\ \frac{w(d-x)}{d-c}, & c \leq x \leq d, \\ 0, & \text { otherwise, }\end{cases}
$$




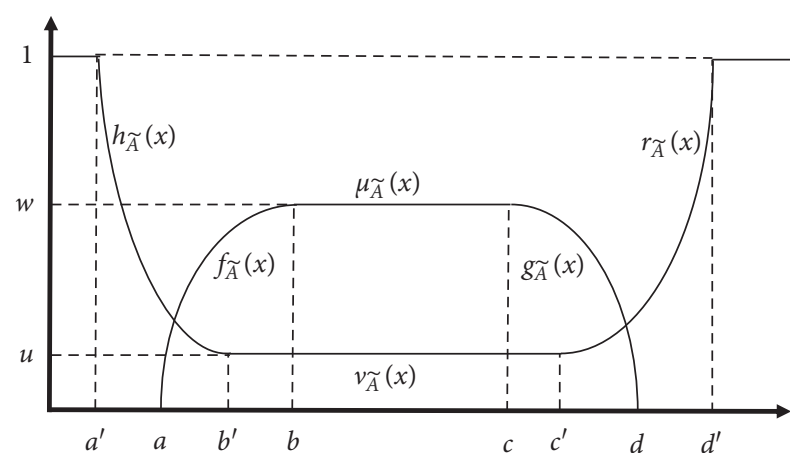

Figure 1: An IFN $\widetilde{A}=\left(\mu_{\widetilde{A}}, v_{\widetilde{A}}\right)$.

$$
v_{\widetilde{A}}(x)= \begin{cases}\frac{(u-1)\left(x-a^{\prime}\right)}{b^{\prime}-a^{\prime}}+1, & a^{\prime} \leq x \leq b^{\prime}, \\ u, & b^{\prime} \leq x \leq c^{\prime}, \\ \frac{(u-1)\left(x-d^{\prime}\right)}{c^{\prime}-d^{\prime}}+1, & c^{\prime} \leq x \leq d^{\prime}, \\ 1, & \text { otherwise. }\end{cases}
$$

We denote a TraIFN $\tilde{A}$ by $\widetilde{A}=\left\langle(a, b, c, d ; w),\left(a^{\prime}\right.\right.$, $\left.\left.b^{\prime}, c^{\prime}, d^{\prime} ; u\right)\right\rangle$ (Figure 2).

In Definition 5, if $w=1$ and $u=0$, then, we have a normal TraIFN. Also, TriIFN is a special case of TraIFN in which $b=c$ and $b^{\prime}=c^{\prime}$.

Figure 3 shows the hesitation degree of belonging or not belonging an element $x$ to TraIFN $\widetilde{A}$.

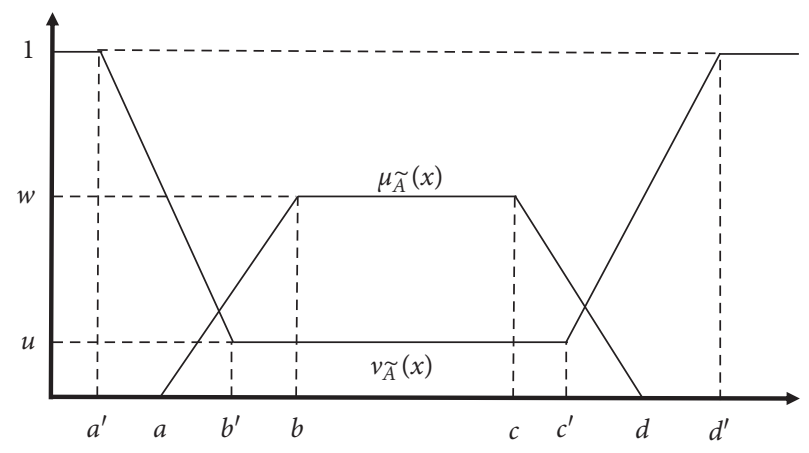

Figure 2: A TraIFN $\widetilde{A}=\left\langle(a, b, c, d ; w),\left(a^{\prime}, b^{\prime}, c^{\prime}, d^{\prime} ; u\right)\right\rangle$.

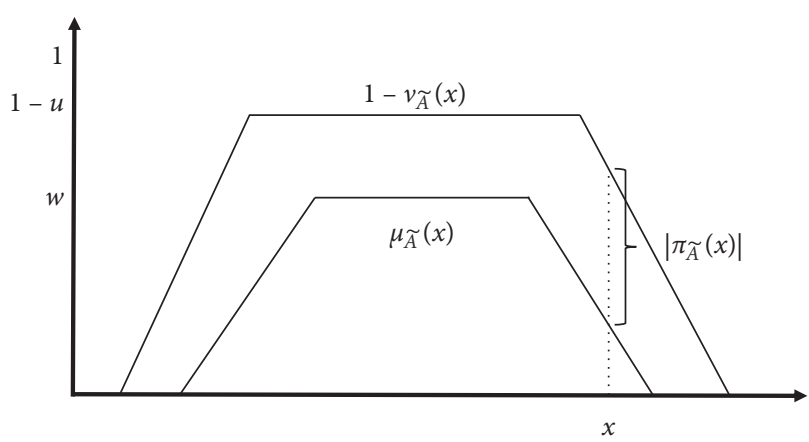

FIGURE 3: Hesitation degree of element $x\left(\pi_{\widetilde{A}}(x)=1-\mu_{\widetilde{A}}(x)-\right.$ $\left.v_{\widetilde{A}}(x)\right)$.

By Definition $4, \alpha$-cut, $\beta$-cut, and $(\alpha, \beta)$-cut sets of a TraIFN $\widetilde{A}=\left\langle(a, b, c, d ; w),\left(a^{\prime}, b^{\prime}, c^{\prime}, d^{\prime} ; u\right)\right\rangle$ are the following closed intervals, respectively,

$$
\begin{aligned}
\widetilde{A}_{\alpha} & =\left\{x \mid \mu_{\widetilde{A}}(x) \geq \alpha\right\}=\left[a+\frac{\alpha}{w}(b-a), d+\frac{\alpha}{w}(c-d)\right], \\
\widetilde{A}_{\beta} & =\left\{x \mid v_{\widetilde{A}}(x) \leq \beta\right\}=\left[a^{\prime}+\frac{1-\beta}{1-u}\left(b^{\prime}-a^{\prime}\right), d^{\prime}+\frac{1-\beta}{1-u}\left(c^{\prime}-d^{\prime}\right)\right], \\
\widetilde{A}_{\alpha, \beta} & =\left\{x \mid \mu_{\widetilde{A}}(x) \geq \alpha, v_{\widetilde{A}}(x) \leq \beta\right\}, \\
& =\left[a+\frac{\alpha}{w}(b-a), d+\frac{\alpha}{w}(c-d)\right] \cap\left[a^{\prime}+\frac{1-\beta}{1-u}\left(b^{\prime}-a^{\prime}\right), d^{\prime}+\frac{1-\beta}{1-u}\left(c^{\prime}-d^{\prime}\right)\right] .
\end{aligned}
$$

We can see that the $(\alpha)$-cut set of a TraIFN $\widetilde{A}$ can be calculated from the intersection of the closed intervals obtained from the $\alpha$-cut and $\beta$-cut sets of $\widetilde{A}$. These three cut sets $(\alpha$-cut, $\beta$-cut, and $(\alpha, \beta)$-cut sets) remove some of the elements of a given TraIFN, whose degrees of its membership and nonmembership functions do not satisfy the levels given as arguments of the corresponding operators.

Definition 6 (see [25]). Let $\widetilde{A}_{1}=\left\langle\left(a_{1}, b_{1}, c_{1}, d_{1} ; w_{1}\right),\left(a_{1}^{\prime}, b_{1}^{\prime}\right.\right.$, $\left.\left.c_{1}^{\prime}, d_{1}^{\prime} ; u_{1}\right)\right\rangle$ and $\widetilde{A}_{2}=\left(a_{2}, b_{2}, c_{2}, d_{2} ; w_{2}\right),\left(a_{2}^{\prime}, b_{2}^{\prime}, c_{2}^{\prime}, d_{2}^{\prime} ; u_{2}\right)$ be 
two arbitrary TraIFNs and $\lambda \in \mathbb{R}$; then, the arithmetic operations are stipulated as follows:

$$
\begin{aligned}
\widetilde{A}_{1} \oplus \widetilde{A}_{2} & =\left\langle\left(a_{1}+a_{2}, b_{1}+b_{2}, c_{1}+c_{2}, d_{1}+d_{2} ; \min \left\{w_{1}, w_{2}\right\}\right),\left(a_{1}^{\prime}+a_{2}^{\prime}, b_{1}^{\prime}+b_{2}^{\prime}, c_{1}^{\prime}+c_{2}^{\prime}, d_{1}^{\prime}+d_{2}^{\prime} ; \max \left\{u_{1}, u_{2}\right\}\right)\right\rangle, \\
\widetilde{A}_{1} \ominus \widetilde{A}_{2} & =\left\langle\left(a_{1}-d_{2}, b_{1}-c_{2}, c_{1}-b_{2}, d_{1}-a_{2} ; \min \left\{w_{1}, w_{2}\right\}\right),\left(a_{1}^{\prime}-d_{2}^{\prime}, b_{1}^{\prime}-c_{2}^{\prime}, c_{1}^{\prime}-b_{2}^{\prime}, d_{1}^{\prime}-a_{2}^{\prime} ; \max \left\{u_{1}, u_{2}\right\}\right)\right\rangle, \\
\lambda \widetilde{A}_{1} & = \begin{cases}\left\langle\left(\lambda a_{1}, \lambda b_{1}, \lambda c_{1}, \lambda d_{1} ; w_{1}\right),\left(\lambda a_{1}^{\prime}, \lambda b_{1}^{\prime}, \lambda c_{1}^{\prime}, \lambda d_{1}^{\prime} ; u_{1}\right)\right\rangle, & \text { if } \lambda \geq 0, \\
\left\langle\left(\lambda d_{1}, \lambda c_{1}, \lambda b_{1}, \lambda a_{1} ; w_{1}\right),\left(\lambda d_{1}^{\prime}, \lambda c_{1}^{\prime}, \lambda b_{1}^{\prime}, \lambda a_{1}^{\prime} ; u_{1}\right)\right\rangle, & \text { if } \lambda<0 .\end{cases}
\end{aligned}
$$

Ma et al. [52] proposed a new approach for defining a fuzzy number. In this approach, the variable on the vertical axis in Figure 1 is considered as an independent variable. By generalizing this approach, we obtain the following definition for IFNs.

Definition 7. $\widetilde{A}=\left(\mu_{\widetilde{A}}^{\prime}, v_{\widetilde{A}}^{\prime}\right)$ is an IFN, in which $\mu_{\widetilde{A}}^{\prime}$ is a pair $(\underline{m}(r), \bar{m}(r))$ of functions $\underline{m}(r)$ and $\bar{m}(r) ; 0 \leq r \leq w$ which satisfy the following requirements:

(1) $\underline{m}(r)$ is a bounded monotonic increasing left continuous function

(2) $\bar{m}(r)$ is a bounded monotonic decreasing left continuous function

(3) $\underline{m}(r) \leq \bar{m}(r), 0 \leq r \leq w$,

and $v_{\widetilde{A}}^{\prime}$ is a pair $(\underline{n}(r), \bar{n}(r))$ of functions $\underline{n}(r)$ and $\bar{n}(r) ; u \leq \quad r \leq 1$ which satisfy the following requirements:

(1) $\underline{n}(r)$ is a bounded monotonic decreasing left continuous function

(2) $\bar{n}(r)$ is a bounded monotonic increasing left continuous function

(3) $\underline{n}(r) \leq \bar{n}(r), u \leq r \leq 1$

So, by Definition 7 , we can represent a TraIFN as $\widetilde{A}=\left(\mu_{\widetilde{A}}^{\prime}, v_{\widetilde{A}}^{\prime}\right)$, where $\mu_{\widetilde{A}}^{\prime}(r)=(\underline{m}(r), \bar{m}(r))=(a+(r / w)(b-$ $a), d+(r / w)(c-d)), 0 \leq r \leq w$, and $v_{\widetilde{A}}^{\prime}(r)=(\underline{n}(r), \bar{n}(r))=$ $\left(a^{\prime}+((1-r) /(1-u))\left(b^{\prime}-a^{\prime}\right), d^{\prime}+((1-r) /(1-u))\left(c^{\prime}-\right.\right.$ $\left.\left.d^{\prime}\right)\right), u \leq r \leq 1$ (Figure 4).

As mentioned above, in Definition 7, the variable on the vertical axis is considered as an independent variable. It provides the ability to obtain the location of the left and right parts of the membership and nonmembership functions of an IFN on the horizontal axis for different amounts of the independent variable. In the following, we will use this property to assign an appropriate parametric index to IFNs.

\section{Ranking Intuitionistic Fuzzy Numbers (IFNs)}

In real-world applications, dealing with imprecise information is a common problem, and the uncertainty is unavoidably involved in every real-world problem. To model these problems, we should correctly incorporate the uncertainty concept into the problem description. Because of the ability of the intuitionistic fuzzy numbers to express imprecise information (by applying the hesitation concept), they are a useful tool to model these problems. One of the first important issues after the definition of intuitionistic fuzzy numbers is how to compare and rank them. The comparison and ranking of intuitionistic fuzzy numbers are complicated, and different methods have been proposed to address this problem.

Engaging the decision maker in the decision process provides flexibility and is a fascinating phenomenon [24]. Using $\alpha$-cuts, Shureshjani and Darehmiraki [39] proposed a parametric method to rank fuzzy numbers based on the decision maker's opinion. In addition to $\alpha$-cuts, we have another important concept in IFSs, namely, hesitation. Hesitation in an IFN, as shown in Figure 3, is an area between "one minus nonmembership function" and "membership function." It is clear that, optimally, this area will be added to the membership function, and in the most pessimistic view, we should consider this area as a part of the nonmembership function. Thus, in ranking IFNs, it would be appropriate to consider a degree of optimism (or pessimism) on the part of the decision maker in the decisionmaking process.

In this section, to consider the abovementioned points, we generalize Shureshjani and Darehmiraki's method [39] and propose a new parametric method for ranking IFNs based on the decision level and hesitation degree parameters.

3.1. The Proposed Index. For an arbitrary IFN $\widetilde{A}=\left(\mu_{\widetilde{A}}^{\prime}, v_{\widetilde{A}}^{\prime}\right)$, we assign the following index:

$$
\begin{aligned}
& S_{\alpha, k}(\widetilde{A})=\int_{\alpha}^{w}(\underline{m}(r)+\bar{m}(r)) \mathrm{d} r+k\left[\int_{\alpha}^{1-u}\left(\underline{n}(r)+\overline{n^{\prime}}(r)\right) \mathrm{d} r\right. \\
& \left.-\int_{\alpha}^{w}(\underline{m}(r)+\bar{m}(r)) \mathrm{d} r\right], \quad 0 \leq \alpha<w, k \in[0,1],
\end{aligned}
$$

where $\underline{n}^{\prime}(r)$ and $\overline{n^{\prime}}(r)$ belong to $1-v_{\widetilde{A}}^{\prime}(r)$.

The selection of $\alpha$ and $k$ parameters depend on the decision maker's idea. From the $S_{\alpha, k}$ index definition, we can see that, after the selection of $\alpha$ parameter, only the elements of an IFN with the membership and hesitation values of larger than or equal to $\alpha$ will be important. Therefore, if we choose $\alpha$ parameter close to one, we have a "high-level decision," and a "low-level decision" is made when the selected $\alpha$ is close to zero. 


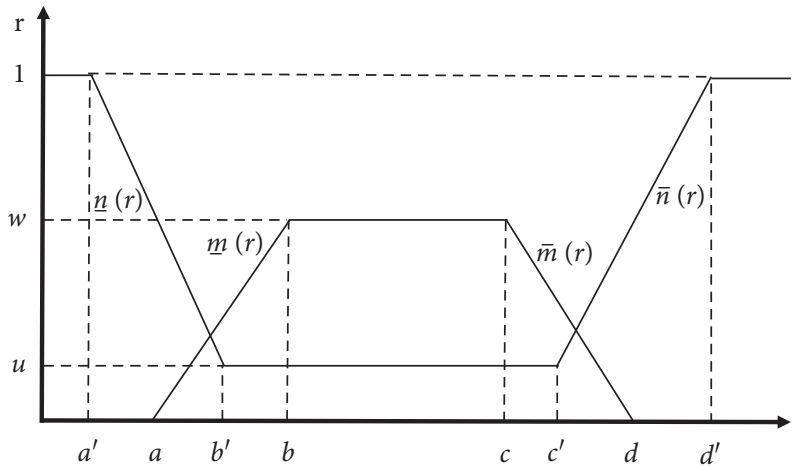

FIgURE 4: Representation of a TraIFN by Definition 7 .

If we rewrite the abovementioned formula, we will have

$$
\begin{aligned}
S_{\alpha, k}(\widetilde{A})= & (1-k) \int_{\alpha}^{w}(\underline{m}(r)+\bar{m}(r)) \mathrm{d} r \\
& +k \int_{\alpha}^{1-u}\left(\underline{n}^{\prime}(r)+\overline{n^{\prime}}(r)\right) \mathrm{d} r .
\end{aligned}
$$

It is clear that if the decision maker sets $k=0$, we have a pessimistic decision because we have not considered the hesitation area in our decision (hesitation area is added to the nonmembership function). Also, if the decision maker sets $k=1$, an optimistic decision has been made because all the hesitation areas are added to the membership function. It should be noted that any choice of $k(0<k<1)$ reflects the desirable hesitation degree that is considered by the decision maker in ranking IFNs process.

For example, for a TraIFN $\widetilde{A}=\left(\mu_{\widetilde{A}}^{\prime}, v_{\widetilde{A}}^{\prime}\right), \underline{m}(r), \bar{m}(r)$, $\underline{n}^{\prime}(r)$, and $\overline{n^{\prime}}(r)$ are represented in Figure 5 .

Let $\widetilde{A}=(a, b, c, d ; w),\left(a^{\prime}, b^{\prime}, c^{\prime}, d^{\prime} ; u\right)$ be a TraIFN; then, we have

$$
\begin{aligned}
\int_{\alpha}^{w}(\underline{m}(r)+\bar{m}(r)) \mathrm{d} r & =\int_{\alpha}^{w}\left(a+\frac{r}{w}(b-a)+d+\frac{r}{w}(c-d)\right) \mathrm{d} r=(a+d)(w-\alpha)+(b+c-a-d)\left(\frac{w^{2}-\alpha^{2}}{2 w}\right) \\
\int_{\alpha}^{1-u}\left(\underline{n}(r)+\overline{n^{\prime}}(r)\right) \mathrm{d} r & =\int_{\alpha}^{1-u}\left(a^{\prime}+\frac{r}{1-u}\left(b^{\prime}-a^{\prime}\right)+d^{\prime}+\frac{r}{1-u}\left(c^{\prime}-d^{\prime}\right)\right) \mathrm{d} r \\
& =\left(a^{\prime}+d^{\prime}\right)(1-u-\alpha)+\left(b^{\prime}+c^{\prime}-a^{\prime}-d^{\prime}\right) \frac{(1-u)^{2}-\alpha^{2}}{2(1-u)}
\end{aligned}
$$

So, the assigned $S_{\alpha, k}$ index to a TraIFN will be as follows:

$$
\begin{aligned}
S_{\alpha, k}(\tilde{A})= & \int_{\alpha}^{w}(\underline{m}(r)+\bar{m}(r)) \mathrm{d} r+k\left[\int_{\alpha}^{1-u}\left(\underline{n}^{\prime}(r)+\overline{n^{\prime}}(r)\right) \mathrm{d} r-\int_{\alpha}^{w}(\underline{m}(r)+\bar{m}(r)) \mathrm{d} r\right], \\
= & (1-k) \int_{\alpha}^{w}(\underline{m}(r)+\bar{m}(r)) \mathrm{d} r+k \int_{\alpha}^{1-u}\left(\underline{n}^{\prime}(r)+\overline{n^{\prime}}(r)\right) \mathrm{d} r \\
= & (1-k)\left[(a+d)(w-\alpha)+(b+c-a-d)\left(\frac{w^{2}-\alpha^{2}}{2 w}\right)\right] \\
& +k\left[\left(a^{\prime}+d^{\prime}\right)(1-u-\alpha)+\left(b^{\prime}+c^{\prime}-a^{\prime}-d^{\prime}\right) \frac{(1-u)^{2}-\alpha^{2}}{2(1-u)}\right], \quad 0 \leq \alpha<w, k \in[0,1] .
\end{aligned}
$$

Figure 6 shows the geometric meaning of the $S_{\alpha, k}$ index for an arbitrary TraIFN $\widetilde{A}$ with $k=0$ and $k=1$, respectively.
We can see that the value of the $S_{\alpha, k}$ index is the summation of the marked areas with solid and empty dots. 


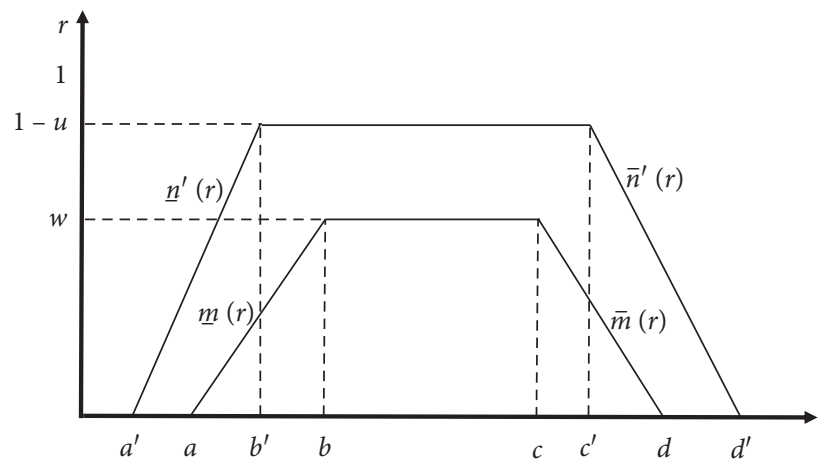

FIgURE 5: $\underline{m}(r), \bar{m}(r), \underline{n}^{\prime}(r)$, and $\overline{n^{\prime}}(r)$ of a TraIFN $\tilde{A}$.

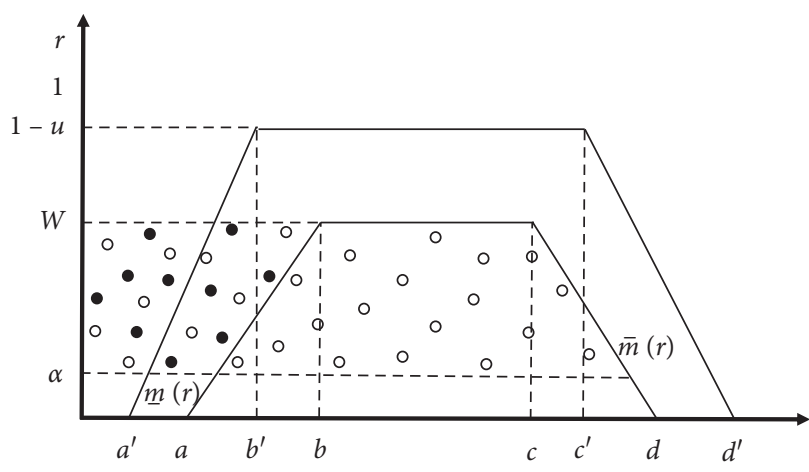

(a)

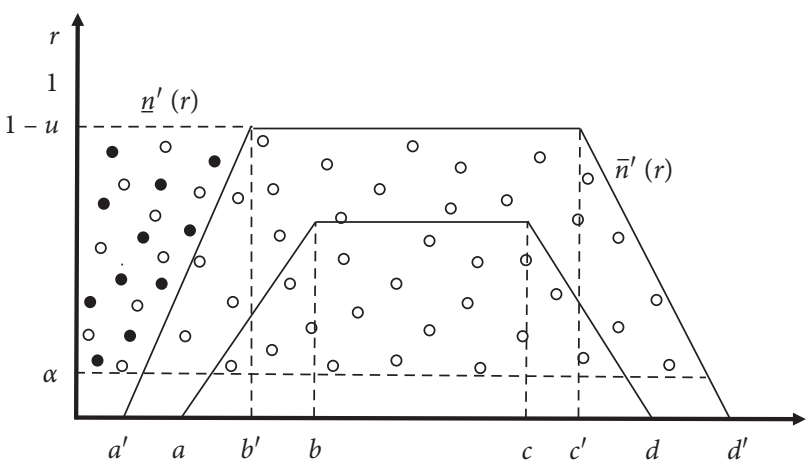

(b)

FIgURE 6: The geometric representation of $S_{\alpha, k}$ index with $k=0$ (a) and $k=1$ (b).

The following theorems demonstrate the reasonable behavior of the proposed index for specific types of IFNs.

$$
S_{\alpha, k}(\widetilde{B})=-S_{\alpha, k}(\widetilde{A})
$$

Theorem 1. If $\widetilde{A}=\left\langle(a, b, c, d ; w),\left(a^{\prime}, b^{\prime}, c^{\prime}, d^{\prime} ; u\right)\right\rangle$ and $\widetilde{B}=\left\langle(-d,-c,-b,-a ; w),\left(-d^{\prime},-c^{\prime},-b^{\prime},-a^{\prime} ; u\right)\right\rangle$, then,

$$
\begin{aligned}
S_{\alpha, k}(\widetilde{B}) & =(1-k)\left[(-d-a)(w-\alpha)+(-c-b+d+a)\left(\frac{w^{2}-\alpha^{2}}{2 w}\right)\right]+k\left[\left(-d^{\prime}-a^{\prime}\right)(1-u-\alpha)+\left(-c^{\prime}-b^{\prime}+d^{\prime}+a^{\prime}\right) \frac{(1-u)^{2}-\alpha^{2}}{2(1-u)}\right] \\
& =(1-k)\left[-(d+a)(w-\alpha)-(c+b-d-a)\left(\frac{w^{2}-\alpha^{2}}{2 w}\right)\right]+k\left[-\left(d^{\prime}+a^{\prime}\right)(1-u-\alpha)-\left(c^{\prime}+b^{\prime}-d^{\prime}-a^{\prime}\right) \frac{(1-u)^{2}-\alpha^{2}}{2(1-u)}\right], \\
& =-(1-k)\left[(d+a)(w-\alpha)+(c+b-d-a)\left(\frac{w^{2}-\alpha^{2}}{2 w}\right)\right]-k\left[\left(d^{\prime}+a^{\prime}\right)(1-u-\alpha)+\left(c^{\prime}+b^{\prime}-d^{\prime}-a^{\prime}\right) \frac{(1-u)^{2}-\alpha^{2}}{2(1-u)}\right] \\
& =-S_{\alpha, k}(\widetilde{A}) .
\end{aligned}
$$

Theorem 2. Let $\widetilde{A}_{1}=\left\langle\left(a_{1}, b_{1}, c_{1}, d_{1} ; w_{1}\right),\left(a_{1}^{\prime}, b_{1}^{\prime}, c_{1}^{\prime}, d_{1}^{\prime} ; u_{1}\right)\right\rangle$ and $\widetilde{A}_{2}=\left\langle\left(a_{2}, b_{2}, c_{2}, d_{2} ; w_{2}\right),\left(a_{2}^{\prime}, b_{2}^{\prime}, c_{2}^{\prime}, d_{2}^{\prime} ; u_{2}\right)\right\rangle$ be two arbitrary TraIFNs where $w_{1}=w_{2}$ and $u_{1}=u_{2}$; then, we have

$$
S_{\alpha, k}\left(\widetilde{A}_{1} \oplus \widetilde{A}_{2}\right)=S_{\alpha, k}\left(\widetilde{A}_{1}\right)+S_{\alpha, k}\left(\widetilde{A}_{2}\right)
$$

Proof. From Definition 6, we have 


$$
\begin{aligned}
& \widetilde{A}_{1} \oplus \widetilde{A}_{2}=\left\langle\left(a_{1}+a_{2}, b_{1}+b_{2}, c_{1}+c_{2}, d_{1}+d_{2} ; w_{1}\right),\left(a_{1}^{\prime}+a_{2}^{\prime}, b_{1}^{\prime}+b_{2}^{\prime}, c_{1}^{\prime}+c_{2}^{\prime}, d_{1}^{\prime}+d_{2}^{\prime} ; u_{1}\right)\right\rangle, \\
S_{\alpha, k}\left(\widetilde{A}_{1} \oplus \widetilde{A}_{2}\right)= & (1-k)\left[\left(\left(a_{1}+a_{2}\right)+\left(d_{1}+d_{2}\right)\right)\left(w_{1}-\alpha\right)+\left(\left(b_{1}+b_{2}\right)+\left(c_{1}+c_{2}\right)-\left(a_{1}+a_{2}\right)-\left(d_{1}+d_{2}\right)\right)\left(\frac{w_{1}^{2}-\alpha^{2}}{2 w_{1}}\right)\right] \\
& +k\left[\left(\left(a_{1}^{\prime}+a_{2}^{\prime}\right)+\left(d_{1}^{\prime}+d_{2}^{\prime}\right)\right)\left(1-u_{1}-\alpha\right)+\left(\left(b_{1}^{\prime}+b_{2}^{\prime}\right)+\left(c_{1}^{\prime}+c_{2}^{\prime}\right)-\left(a_{1}^{\prime}+a_{2}^{\prime}\right)-\left(d_{1}^{\prime}+d_{2}^{\prime}\right)\right) \frac{(1-u)^{2}-\alpha^{2}}{2\left(1-u_{1}\right)}\right], \\
= & \left\{(1-k)\left[\left(a_{1}+d_{1}\right)\left(w_{1}-\alpha\right)+\left(b_{1}+c_{1}-a_{1}-d_{1}\right)\left(\frac{w_{1}^{2}-\alpha^{2}}{2 w_{1}}\right)\right]\right. \\
& \left.+k\left[\left(a_{1}^{\prime}+d_{1}^{\prime}\right)\left(1-u_{1}-\alpha\right)+\left(b_{1}^{\prime}+c_{1}^{\prime}-a_{1}^{\prime}-d_{1}^{\prime}\right) \frac{(1-u)^{2}-\alpha^{2}}{2\left(1-u_{1}\right)}\right]\right\} \\
& +\left\{(1-k)\left[\left(a_{2}+d_{2}\right)\left(w_{1}-\alpha\right)+\left(b_{2}+c_{2}-a_{2}-d_{2}\right)\left(\frac{w_{1}^{2}-\alpha^{2}}{2 w_{1}}\right)\right]\right. \\
& \left.+k\left[\left(a_{2}^{\prime}+d_{2}^{\prime}\right)\left(1-u_{1}-\alpha\right)+\left(b_{2}^{\prime}+c_{2}^{\prime}-a_{2}^{\prime}-d_{2}^{\prime}\right) \frac{(1-u)^{2}-\alpha^{2}}{2\left(1-u_{1}\right)}\right]\right\} \\
= & S_{\alpha, k}\left(\widetilde{A}_{1}\right)+S_{\alpha, k}\left(\widetilde{A}_{2}\right) .
\end{aligned}
$$

Theorem 3. Let $\widetilde{A}=\left\langle(a, b, c, d ; w),\left(a^{\prime}, b^{\prime}, c^{\prime}, d^{\prime} ; u\right)\right\rangle$ and $\lambda \in \mathbb{R}$; then, we have

$$
S_{\alpha, k}(\lambda \widetilde{A})=\lambda S_{\alpha, k}(\widetilde{A}) .
$$

$$
\lambda \widetilde{A}= \begin{cases}\left\langle(\lambda a, \lambda b, \lambda c, \lambda d ; w),\left(\lambda a^{\prime}, \lambda b^{\prime}, \lambda c^{\prime}, \lambda d^{\prime} ; u\right)\right\rangle, & \lambda>0, \\ \left\langle(\lambda d, \lambda c, \lambda b, \lambda a ; w),\left(\lambda d^{\prime}, \lambda c^{\prime}, \lambda b^{\prime}, \lambda a^{\prime} ; u\right)\right\rangle, & \lambda<0 .\end{cases}
$$

Let $\lambda \geq 0$; then, we have

Proof. From Definition 6, we have

$$
\begin{aligned}
\lambda \widetilde{A}= & \left\langle(\lambda a, \lambda b, \lambda c, \lambda d ; w),\left(\lambda a^{\prime}, \lambda b^{\prime}, \lambda c^{\prime}, \lambda d^{\prime} ; u\right)\right\rangle \\
S_{\alpha, k}(\lambda \widetilde{A})= & (1-k)\left[(\lambda a+\lambda d)(w-\alpha)+(\lambda b+\lambda c-\lambda a-\lambda d)\left(\frac{w^{2}-\alpha^{2}}{2 w}\right)\right] \\
& +k\left[\left(\lambda a^{\prime}+\lambda d^{\prime}\right)(1-u-\alpha)+\left(\lambda b^{\prime}+\lambda c^{\prime}-\lambda a^{\prime}-\lambda d^{\prime}\right) \frac{(1-u)^{2}-\alpha^{2}}{2(1-u)}\right], \\
= & \lambda(1-k)\left[(a+d)(w-\alpha)+(b+c-a-d)\left(\frac{w^{2}-\alpha^{2}}{2 w}\right)\right] \\
& +\lambda k\left[\left(a^{\prime}+d^{\prime}\right)(1-u-\alpha)+\left(b^{\prime}+c^{\prime}-a^{\prime}-d^{\prime}\right) \frac{(1-u)^{2}-\alpha^{2}}{2(1-u)}\right] \\
= & \lambda S_{\alpha, k}(\tilde{A}) .
\end{aligned}
$$

Now, if $\lambda<0$, then, we have 


$$
\begin{aligned}
\lambda \widetilde{A}= & \left\langle\left(\lambda d, \lambda c, \lambda b, \lambda a ; w_{1}\right),\left(\lambda d^{\prime}, \lambda c^{\prime}, \lambda b^{\prime}, \lambda a^{\prime} ; u_{1}\right)\right\rangle, \\
S_{\alpha, k}(\lambda \widetilde{A})= & (1-k)\left[(\lambda d+\lambda a)(w-\alpha)+(\lambda c+\lambda b-\lambda d-\lambda a)\left(\frac{w^{2}-\alpha^{2}}{2 w}\right)\right] \\
& +k\left[\left(\lambda d^{\prime}+\lambda a^{\prime}\right)(1-u-\alpha)+\left(\lambda c^{\prime}+\lambda b^{\prime}-\lambda d^{\prime}-\lambda a^{\prime}\right) \frac{(1-u)^{2}-\alpha^{2}}{2(1-u)}\right], \\
= & \lambda(1-k)\left[(d+a)(w-\alpha)+(c+b-d-a)\left(\frac{w^{2}-\alpha^{2}}{2 w}\right)\right] \\
& +\lambda k\left[\left(d^{\prime}+a^{\prime}\right)(1-u-\alpha)+\left(c^{\prime}+b^{\prime}-d^{\prime}-a^{\prime}\right) \frac{(1-u)^{2}-\alpha^{2}}{2(1-u)}\right], \\
= & \lambda S_{\alpha, k}(\widetilde{A}) .
\end{aligned}
$$

Corollary 1. If $\widetilde{A}=\langle(0,0,0,0 ; w),(0,0,0,0 ; u)\rangle$ and $\widetilde{B}=\langle(a, a, a, a ; w),(a, a, a, a ; u)\rangle$, then, we have

$$
\begin{aligned}
& S_{\alpha, k}(\widetilde{A})=0, \\
& S_{\alpha, k}(\widetilde{B})=(1-k)(2 a)(w-\alpha)+k(2 a)(1-u-\alpha), \\
& 0 \leq \alpha<w, k \in[0,1] .
\end{aligned}
$$

3.2. Our Proposed Ranking Approach. Based on the proposed $S_{\alpha, k}$ index, we can give the following definitions to rank IFNs.

Definition 8. Let $\widetilde{A}=\left(\mu \frac{\prime}{\widetilde{A}}, v_{\widetilde{A}}^{\prime}\right)$ and $\widetilde{B}=\left(\mu \frac{1}{\widetilde{B}}, v_{\widetilde{B}}^{\prime}\right)$ be two arbitrary IFNs; then, for a decision level higher than $\alpha$ and a selected hesitation degree $k \quad\left(0 \leq \alpha<\min \left\{w_{\widetilde{A}}, w_{\widetilde{B}}\right\}\right.$, $k \in[0,1])$, we have
(1) $\widetilde{A} \prec_{\alpha, k} \widetilde{B} \longleftrightarrow S_{\alpha, k}(\widetilde{A})<S_{\alpha, k}(\widetilde{B})$,
(2) $\widetilde{A} \sim{ }_{\alpha, k} \widetilde{B} \longleftrightarrow S_{\alpha, k}(\widetilde{A})=S_{\alpha, k}(\widetilde{B})$,
(3) $\widetilde{A}>_{\alpha, k} \widetilde{B} \longleftrightarrow S_{\alpha, k}(\widetilde{A})>S_{\alpha, k}(\widetilde{B})$.

Definition 9. Let $\widetilde{A}=\left(\mu \frac{\prime}{\widetilde{A}}, v_{\widetilde{A}}^{\prime}\right)$ and $\widetilde{B}=\left(\mu_{\widetilde{B}}^{\prime}, v_{\widetilde{B}}^{\prime}\right)$ be two arbitrary IFNs; then, by considering $(0 \leq \alpha<$ $\left.\min \left\{w_{\widetilde{A}}, w_{\widetilde{B}}\right\}, k \in[0,1]\right)$, we have

(1) $\widetilde{A} \prec \widetilde{B} \longleftrightarrow \forall \alpha, k: S_{\alpha, k}(\widetilde{A})<S_{\alpha, k}(\widetilde{B})$,

(2) $\widetilde{A} \sim \widetilde{B} \longleftrightarrow \forall \alpha, k: S_{\alpha, k}(\widetilde{A})=S_{\alpha, k}(\widetilde{B})$,

(3) $\widetilde{A} \succ \widetilde{B} \longleftrightarrow \forall \alpha, k: S_{\alpha, k}(\widetilde{A})>S_{\alpha, k}(\widetilde{B})$.

Wang and Kerre [51] suggested seven reasonable properties to evaluate the rationality of the proposed indices for ranking fuzzy numbers. Here, we examine the validity of our proposed index for ranking IFNs based on Wang and Kerre's [51] approach.

Let $I$ be the set of intuitionistic fuzzy quantities and $\mathscr{A}$ be a finite subset of $I$.

Property 1. For an arbitrary finite subset $\mathscr{A}$ of $I$ and $\widetilde{A} \in \mathscr{A}$, $\widetilde{A} \lesssim \widetilde{A}(\widetilde{A}$ has at least the same ranking as $\widetilde{A})$ by the proposed ranking method on $\mathscr{A}$.
Proof. After calculating the $S_{\alpha, k}(\widetilde{A})$, it is clear that we have $\underset{\sim}{\forall}, k: \underset{\widetilde{A}}{S_{\alpha, k}}(\widetilde{A})=S_{\alpha, k}(\widetilde{A})$. So, according to Definition 9, $\widetilde{A} \sim \widetilde{A}$.

Property 2. For an arbitrary finite subset $\mathscr{A}$ of I and $(\widetilde{A}, \widetilde{B}) \in \mathscr{A}^{2}, \widetilde{A} z \widetilde{B}$ and $\widetilde{A} \lesssim \widetilde{B}$ by the proposed ranking method on $\mathscr{A}$, we should have $\widetilde{A} \sim \widetilde{B}$ by the proposed ranking method on $\mathscr{A}$.

Proof. Let $\widetilde{\mathrm{A}} \succsim \widetilde{\mathrm{B}}$ and $\widetilde{\mathrm{A}} \lesssim \widetilde{\mathrm{B}}$; then, simultaneously, we have $\forall \alpha, k: \quad S_{\alpha, k}(\widetilde{A}) \geq S_{\alpha, k}(\widetilde{B})$ and $S_{\alpha, k}(\widetilde{A}) \leq S_{\alpha, k}(\widetilde{B})$ So, $\forall \alpha, k$ : $S_{\alpha, k}(\widetilde{A})=S_{\alpha, k}(\widetilde{B})$ and according to Definition 9, $\widetilde{A} \sim \widetilde{B}$.

Property 3. For an arbitrary finite subset $\mathscr{A}$ of I and $(\widetilde{A}, \widetilde{B}, \widetilde{C}) \in \mathscr{A}^{3}, \widetilde{A} z \widetilde{B}$ and $\widetilde{B} \succsim \widetilde{C}$ by the proposed ranking method on $\mathscr{A}$, we should have $\widetilde{A} \gtrsim \widetilde{C}$ by the proposed ranking method on $\mathscr{A}$.

Proof. From $\widetilde{A} \gtrsim \widetilde{B}$ and $\widetilde{B} \gtrsim \widetilde{C}$, we have $\forall \alpha, k$ : $S_{\alpha, k}(\widetilde{A}) \geq S_{\alpha, k}(\widetilde{B})$ and $S_{\alpha, k}(\widetilde{B}) \geq S_{\alpha, k}(\widetilde{\widetilde{C}})$. These imply that $\forall \alpha, k: S_{\alpha, k}(\widetilde{A}) \geq S_{\alpha, k}(\widetilde{C})$ that means $\widetilde{A} \gtrsim \widetilde{C}$.

Property 4. Let $\widetilde{A}$ and $\widetilde{B}$ be two arbitrary IFNs of an arbitrary finite subset $\mathscr{A}$ of I with $w=w_{\widetilde{A}}=w_{\widetilde{B}}$ and $u=u_{\widetilde{A}}=u_{\widetilde{B}}$, $\inf \sup p\left(\mu_{\widetilde{A}}\right)>\operatorname{supsup} p\left(\mu_{\widetilde{B}}\right)$ and $\inf \operatorname{supp}\left(v_{\widetilde{A}}\right)>\operatorname{supp}\left(v_{\widetilde{B}}\right)$, we should have $\widetilde{A}>\widetilde{B}$ by the proposed ranking method on $\mathscr{A}$.

Proof. Using the $S_{\alpha, k}$ index, we have $S_{\alpha, k}(\widetilde{A})=(1-k)$ $\int_{\alpha}^{w}(\underline{m}(r)+\bar{m}(r)) \mathrm{d} r+k \int_{\alpha}^{1-u} \underline{n}^{\prime}(r)+\overline{n^{\prime}}(r) \mathrm{d} r=(1-k) \int_{\alpha}^{w} 2$. $M_{1}\left(\widetilde{A}_{r}\right) \mathrm{d} r+k \int_{\alpha}^{1-u} 2 \cdot M_{2}\left(\widetilde{A}_{r}\right) \mathrm{d} r \geq(1-k) \int_{\alpha}^{w} 2 \cdot \inf \operatorname{supp}\left(\mu_{\widetilde{A}}\right)$ $\mathrm{d} r+k \int_{\alpha}^{1-u} 2 \cdot \operatorname{infsupp}\left(v_{\widetilde{A}}\right) \mathrm{d} r$, where $M_{1}\left(\widetilde{A}_{r}\right)$ is the mean value of $\alpha$-cut $\widetilde{A}_{r}$ and $M_{2}\left(\widetilde{A}_{r}\right)$ is the mean value of $\beta$-cut $\widetilde{A}_{r}$.

Similarly, $\quad S_{\alpha, k}(\widetilde{B}) \leq(1-k) \int_{\alpha}^{w} 2 \cdot \sup \operatorname{supp}\left(\mu_{\widetilde{B}}\right) \mathrm{d} r+k$ $\int_{\alpha}^{1-u} 2 \cdot \sup \operatorname{supp}\left(v_{\widetilde{B}}\right) \mathrm{d} r$ Therefore, $\forall \alpha, k: S_{\alpha, k}(\widetilde{A})>S_{\alpha, k}(\widetilde{B})$, i.e., $\widetilde{A}>\widetilde{B}$. 
Property 5. Let I and $I^{\prime}$ be two arbitrary finite sets of intuitionistic fuzzy quantities in which the proposed ranking method can be applied and $\widetilde{A}$ and $\widetilde{B}$ are in $I \cap I^{\prime}$. By the proposed ranking method $\widetilde{A} \succ \widetilde{B}$ on $I^{\prime}$ iff $\widetilde{A} \succ \widetilde{B}$ on I.

Proof. Trivial.

Property 6. Let $\widetilde{A}, \widetilde{B}$, and $\widetilde{C}$ are three arbitrary TraIFNs of an arbitrary finite subset $\mathscr{A}$ of I and $(\widetilde{A}, \widetilde{B}, \widetilde{C}) \in \mathscr{A}^{3}$ with $w_{\widetilde{A}}=$ $w_{\widetilde{B}}=w_{\widetilde{C}}$ and $u_{\widetilde{A}}=u_{\widetilde{B}}=u_{\widetilde{C}}$. If $\widetilde{A}>\widetilde{B}$, we should have $\widetilde{A} \oplus \widetilde{C}>\widetilde{B} \oplus \widetilde{C}$ by the proposed ranking method on $\mathscr{A}$.

Proof. Let $\widetilde{A}>\widetilde{B}$, so $\forall \alpha, k$ : $S_{\alpha, k}(\widetilde{A})>S_{\alpha, k}(\widetilde{B})$. From Theorem 2, we have $S_{\alpha, k}(\widetilde{A} \oplus \widetilde{C})=S_{\alpha, k}(\widetilde{A})+S_{\alpha,}, k(\widetilde{C}) \geq S_{\alpha, k}(\widetilde{B})+$ $S_{\alpha, k}(\widetilde{C})=S_{\alpha, k}(\widetilde{B} \oplus \widetilde{C})$, which means $\widetilde{A} \oplus \widetilde{C}>\widetilde{B} \oplus \widetilde{C}$.

Property 7. Let $\widetilde{A}$ and $\widetilde{B}$ are two arbitrary TraIFNs of an arbitrary finite subset $\mathscr{A}$ of I and $(\widetilde{A}, \widetilde{B}) \in \mathscr{A}^{2}, \widetilde{A}>\widetilde{B}$ by the proposed ranking method on $\mathscr{A}$ and $\lambda \in \mathbb{R}$, for $\lambda>0$ we should have $\lambda \widetilde{A}>\lambda \widetilde{B}$ and for $\lambda<0$ we should have $\lambda \widetilde{A}<\lambda \widetilde{B}$ by the proposed ranking method on $\mathscr{A}$.

Proof. $\widetilde{A}>\widetilde{B}$ implies that $\forall \alpha, k: S_{\alpha, k}(\widetilde{A})>S_{\alpha, k}(\widetilde{B})$. From Theorem 3, if $\lambda>0$, we have $S_{\alpha, k}(\lambda \widetilde{A})=\lambda S_{\alpha, k}(\widetilde{A})>$ $\lambda S_{\alpha, k}(\widetilde{B})=S_{\alpha, k}(\lambda \widetilde{B})$ that means $\lambda \widetilde{A}>\lambda \widetilde{B}$. Also, for $\lambda<0$, $S_{\alpha, k}(\lambda \widetilde{A})=\lambda S_{\alpha, k}(\widetilde{A})<\lambda S_{\alpha, k}(\widetilde{B})=S_{\alpha, k}(\lambda \widetilde{B}) \quad$ that means $\lambda \widetilde{A} \prec \lambda \widetilde{B}$.

3.3. The Advantages of the Proposed Method. We demonstrate the advantages of the proposed method over some other approaches by illustrative examples.

In Example 1, we will see that the proposed method can efficiently compare intersected TraIFNs, and the decision maker can apply his/her preferences by choosing appropriate amounts of decision level $(\alpha)$ and hesitation degree (k). Similar to our proposed method, Darehmiraki [38] tried to extend Shureshjani and Daremiraki's [39] method to rank IFNs. However, Example 2 shows that there are some situations where this method led to inappropriate results. As mentioned in the introduction section, various methods have been proposed for ranking IFNs. All these methods can be classified into two categories: parametric and nonparametric. In the parametric approach, using value and ambiguity concepts is the most popular (see e.g. [25-28]). Among these papers, two of the newest are that of Nayagam et al. [28] and Chutia and Saikia [25]. In Example 3, our parametric method is compared with that proposed by Nayagam et al. [28] and Chutia and Saikia [25] and two other nonparametric methods (by Ye [15] and Nayagam et al. [32]).
Example 1. Consider two intersected TraIFNs $\widetilde{A}=\langle(0,1,2,3 ; 0.6),(0,1,2,5 ; 0.1)\rangle \quad$ and $\widetilde{B}=\langle(0,1,2,3$; $0.8),(0,1,2,3 ; 0.1)\rangle . S_{\alpha, k}$ index of $\widetilde{A}$ and $\widetilde{B}$ are calculated as follows: $S_{\alpha, k}(\widetilde{A})=(1-k)[3(0.6-\alpha)]+k[5(0.9-\alpha)-(2 /$ 1.8) $\left.\quad\left(0.81-\alpha^{2}\right)\right] \quad$ and $\quad S_{\alpha, k}(\widetilde{B})=(1-k)[3(0.8-\alpha)]+$ $k[3(0.9-\alpha)],(0 \leq \alpha<0.6, k \in[0,1])$.

In Figure $7, S_{\alpha, k}$ functions of TraIFNs $\widetilde{A}$ and $\widetilde{B}$ are plotted. As can be seen, different decision levels ( $\alpha$-levels) and hesitation degrees $(k)$ led to different ranking results. For example, in an intermediate-level decision $(\alpha=0.5)$, if a low hesitation degree $(k=0)$ is selected by a decision maker, we have $\widetilde{A} \prec_{0.5,0} \widetilde{B}$, but for a high hesitation degree $(k=1)$, we obtain $\widetilde{A}>_{0.5,1} \widetilde{B}$. Also, for a relatively high hesitation degree $(k=0.7)$, if a low-level decision $(\alpha=0)$ is chosen, $\widetilde{A} \succ_{0,0.7} \widetilde{B}$, but for an intermediate-level decision $(\alpha=0.5), \widetilde{A} \prec_{0.5,0.7} \widetilde{B}$. Equality can happen for different amounts of $\alpha$ and $k$ too (see Table 3).

Example 2. Consider two TraIFNs $\widetilde{A}=\langle(2,3,4,5$; $0.8),(2,3,4,5 ; 0.1)\rangle$ and $\widetilde{B}=\langle(7,8,9,10 ; 0.8),(7,8,9,10$; $0.1)\rangle$. It is clear that the acceptable result will be $\widetilde{A} \prec \widetilde{B}$ (see Figure 8).

In an attempt to generalize Shureshjani and Darehmiraki's [39] method to rank IFNs, Darehmiraki [38] proposed an index using $\alpha$ - and $\beta$-cuts. Practically, however, it is evident that this index leads to inappropriate results.

The obtained results from Darehmiraki's [38] method for different amounts of the parameters are presented in Table 4. As observed, the results are not correct.

But from our proposed method, we have

$$
\begin{aligned}
S_{\alpha, k}(\widetilde{A})= & 7[(1-k)(0.8-\alpha)+k(0.9-\alpha)], \\
S_{\alpha, k}(\widetilde{B})= & 17[(1-k)(0.8-\alpha)+k(0.9-\alpha)], \\
& (0 \leq \alpha<0.8, k \in[0,1]) .
\end{aligned}
$$

It is clear that, for all decision levels of $\alpha$ and hesitation degrees of $k$, we have

$$
S_{\alpha, k}(\widetilde{A})<S_{\alpha, k}(\widetilde{B}), \quad 0 \leq \alpha<0.8, k \in[0,1] .
$$

(See Figure 9). So from Definition 9, we obtain $\widetilde{A} \prec \widetilde{B}$ that is a reasonable result.

Example 3. Consider three intersected TraIFNs $\widetilde{A}=\langle(0,0.4$, $0.7,0.8 ; 1),(0,0.3,0.8,0.9 ; 0)\rangle, \quad \widetilde{B}=\langle(0.2,0.5,0.5,0.9 ; 1)$, $(0.2,0.5,0.5,1 ; 0)\rangle$, and $\widetilde{C}=\langle(0,0.6,0.6,0.8 ; 1),(0,0.6$, $0.6,0.9 ; 0)\rangle$.

From the proposed index, we have

$$
\begin{aligned}
& S_{\alpha, k}(\widetilde{A})=(1-k)\left[0.8(1-\alpha)+0.15\left(1-\alpha^{2}\right)\right]+k\left[0.9(1-\alpha)+0.1\left(1-\alpha^{2}\right)\right], \\
& S_{\alpha, k}(\widetilde{B})=(1-k)\left[1.1(1-\alpha)-0.05\left(1-\alpha^{2}\right)\right]+k\left[1.2(1-\alpha)-0.1\left(1-\alpha^{2}\right)\right], \\
& S_{\alpha, k}(\widetilde{C})=(1-k)\left[0.8(1-\alpha)+0.2\left(1-\alpha^{2}\right)\right]+k\left[0.9(1-\alpha)+0.15\left(1-\alpha^{2}\right)\right] .
\end{aligned}
$$




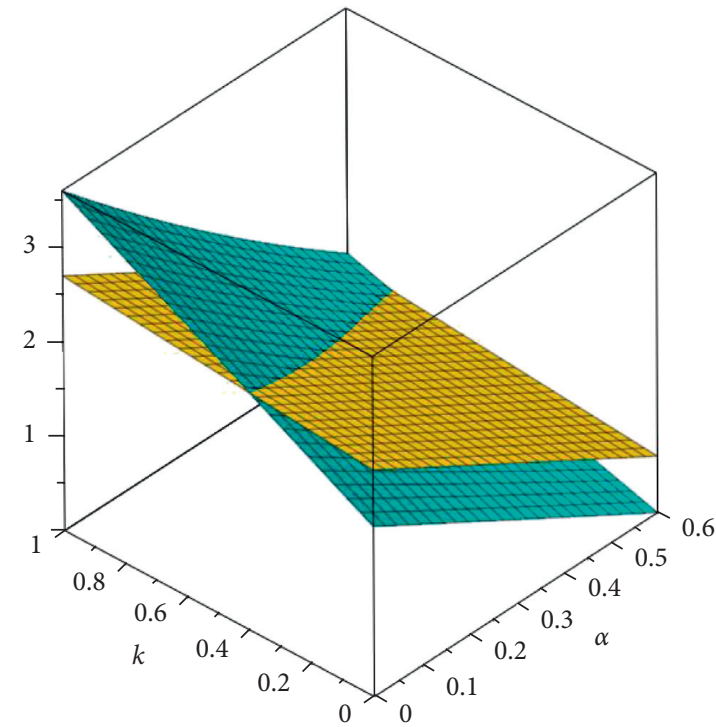

Figure 7: $S_{\alpha, k}$ index of two TraIFNs $\widetilde{A}$ and $\widetilde{B}$.

TABLE 3: Ranking results for some different amounts of $\alpha$ and $k$.

\begin{tabular}{|c|c|c|c|c|}
\hline$\alpha$ & $k$ & $S_{\alpha, k}(\widetilde{A})$ & $S_{\alpha, k}(\widetilde{B})$ & Rank \\
\hline 0.5 & 0 & 0.3 & 0.9 & $\underset{\sim}{\widetilde{A}} \prec_{0.5,0} \underset{\sim}{\widetilde{B}}$ \\
\hline 0.5 & 1 & 1.38 & 1.2 & $\widetilde{A}>_{0.5,1} \widetilde{B}$ \\
\hline 0 & 0.7 & 3.06 & 2.61 & $\widetilde{A} \succ_{0,0.7} \widetilde{B}$ \\
\hline 0.5 & 0.7 & 1.05 & 1.11 & $\widetilde{A} \prec{ }_{0.5,0.7} \widetilde{B}$ \\
\hline 0 & 0.4 & 2.52 & 2.52 & $\underset{\sim}{\widetilde{A}} \sim_{0,0.4} \widetilde{B}$ \\
\hline 0.3 & 0.6 & 1.68 & 1.68 & $\widetilde{A} \sim{ }_{0.3,0.6} \widetilde{B}$ \\
\hline
\end{tabular}

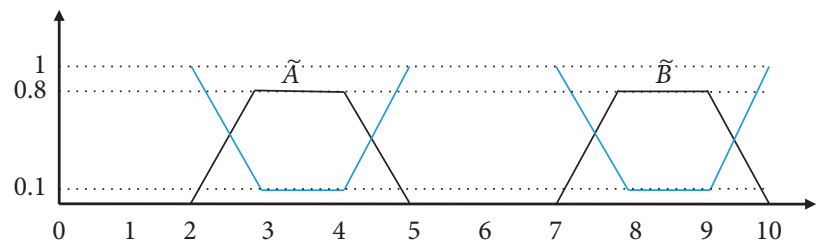

Figure 8 : TraIFNs $\widetilde{A}=\langle(2,3,4,5 ; 0.8),(2,3,4,5 ; 0.1)\rangle$ and $\widetilde{B}=\langle(7,8,9,10 ; 0.8),(7,8,9,10 ; 0.1)\rangle$.

TABle 4: The obtained results from Darehmiraki's [38] method.

\begin{tabular}{lccc}
\hline$\alpha$ and $\beta$ parameters & $Q_{\alpha, \beta}(\widetilde{A})$ & $Q_{\alpha, \beta}(\widetilde{B})$ & Ranking result \\
\hline$\alpha=0.7, \beta=0.3$ & -0.7 & -1.7 & $\widetilde{B}<\widetilde{A}$ \\
$\alpha=0.6, \beta=0.4$ & -0.7 & -1.7 & $\widetilde{B}<\widetilde{A}$ \\
$\alpha=0.7, \beta=0.2$ & 0 & 0 & $\widetilde{B} \sim \widetilde{A}$ \\
\hline
\end{tabular}

These parametric functions are compared and plotted at different decision levels of $\alpha(0 \leq \alpha<1)$ and hesitation degrees of $k(0 \leq k \leq 1)$ in Figure 10 .

As can be seen from Figure 10, for different amounts of $\alpha$ and $k$, we obtain 5 different ranking results as follows:

(1) If $0 \leq \alpha<0.2, k \in[0,1]$, then, $S_{\alpha, k}(\widetilde{A})<S_{\alpha, k}(\widetilde{C})<$ $S_{\alpha, k}(\widetilde{B})$, and from Definition 8 , we have $\widetilde{A} \prec_{\alpha, k} \widetilde{C} \prec_{\alpha, k} \widetilde{B}$
(2) If $\alpha=0.2, k \in[0,1], \quad$ then, $S_{\alpha, k}(\widetilde{A})<S_{\alpha, k}(\widetilde{C})=$ $S_{\alpha, k}(\widetilde{B})$, and so $\widetilde{A} \prec_{\alpha, k} \widetilde{C} \sim{ }_{\alpha, k} \widetilde{B}$

(3) If $0.2<\alpha<0.5, k \in[0,1]$, then, $S_{\alpha, k}(\widetilde{A})<S_{\alpha, k}(\widetilde{B})<$ $S_{\alpha, k}(\widetilde{C})$, and so $\widetilde{A} \prec_{\alpha, k} \widetilde{B} \prec_{\alpha, k} \widetilde{C}$

(4) If $\quad \alpha=0.5, k \in[0,1]: \quad S_{\alpha, k}(\widetilde{A})=S_{\alpha, k}(\widetilde{B})<S_{\alpha, k}(\widetilde{C})$, and so $\widetilde{A} \sim{ }_{\alpha, k} \widetilde{B} \prec_{\alpha, k} \widetilde{C}$

(5) If $0.5<\alpha<1, k \in[0,1]: S_{\alpha, k}(\widetilde{B})<S_{\alpha, k}(\widetilde{A})<S_{\alpha, k}(\widetilde{C})$, and so $\widetilde{B} \prec_{\alpha, k} \widetilde{A} \prec_{\alpha, k} \widetilde{C}$ 


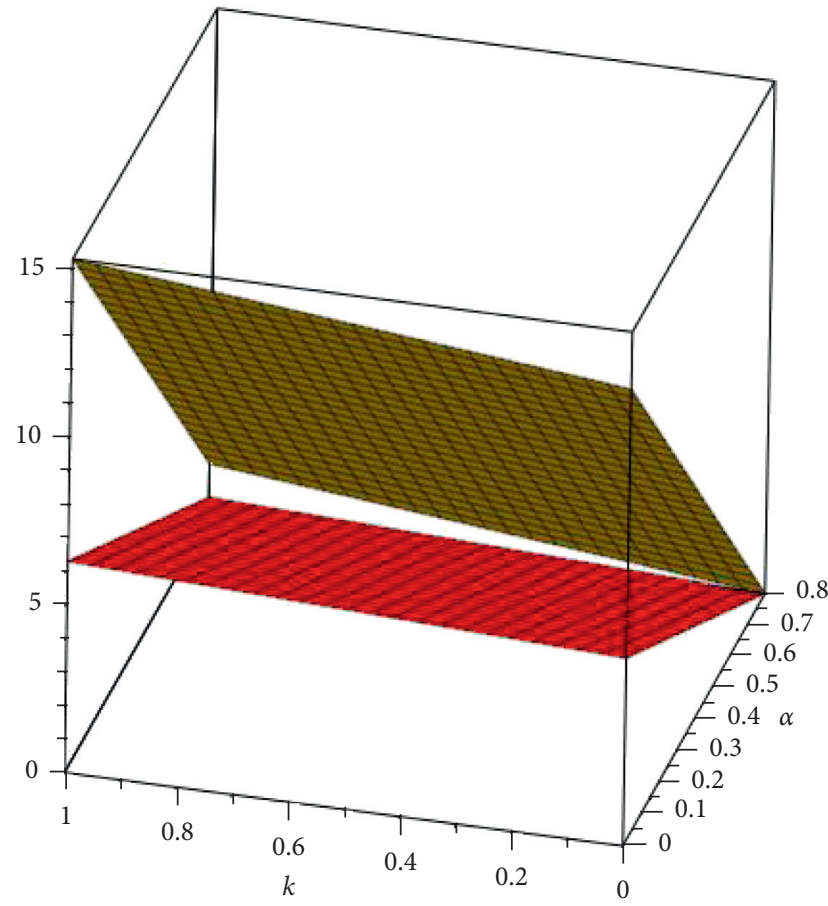

Figure 9: $S_{\alpha, k}$ index of two TraIFNs $\widetilde{A}$ and $\widetilde{B}$.

By applying Chutia and Saikia's [25] method, after plotting value function $\left(V_{\alpha, \beta}\right)$ of $\widetilde{A}, \widetilde{B}$, and $\widetilde{C}$, it can be seen that, for most values of $\alpha$ and $\beta$, we obtain $V_{\alpha, \beta}(\widetilde{A})<V_{\alpha, \beta}(\widetilde{B})$ (see Figure 11 ), so for these values, we have $\widetilde{A} \prec \widetilde{B}$. For specific amounts of $\alpha$ and $\beta$ such as $\alpha=0.8, \beta=0.05$, we have $V_{\alpha, \beta}(\widetilde{B})<V_{\alpha, \beta}(\widetilde{A})$, so $\widetilde{B} \prec \widetilde{A}$ (see Figure 11). Also, for all amounts of $\alpha$ and $\beta$, we obtain $V_{\alpha, \beta}(\tilde{A})<V_{\alpha, \beta}(\widetilde{C})$, so we have $\widetilde{A} \prec \widetilde{C}$ (see Figure 11). Moreover, for different amounts of $\alpha$ and $\beta$, we obtain different ranking results between $\widetilde{B}$ and $\widetilde{C}$ (see Figure 11). So, by appropriate amounts of $\alpha$ and $\beta$, we can obtain the following ranking results:

$$
\begin{aligned}
& \widetilde{A} \prec \widetilde{C} \prec \widetilde{B}, \\
& \widetilde{A} \prec \widetilde{B} \prec \widetilde{C}, \\
& \widetilde{B} \prec \widetilde{A} \prec \widetilde{C} .
\end{aligned}
$$

Ambiguity function $\left(A_{\alpha, \beta}\right)$ of $\widetilde{A}, \widetilde{B}$, and $\widetilde{C}$ is plotted in Figure 12 too. We can see that the equality condition between these IFNs will not occur by Chutia and Saikia's [25] method. Therefore, unlike our method, the ranking results of $\widetilde{A} \prec \widetilde{C} \sim \widetilde{B}$ or $\widetilde{A} \sim \widetilde{B} \prec \widetilde{C}$ are not obtained by Chutia and Saikia's [25] method. Besides, unlike Chutia and Saikia's [25] method that apply a two-level approach to compare and rank IFNs, we assign a parametric index to IFNs which is dependent on the decision maker's idea. Therefore, we can easily apply our method for solving optimization problems under intuitionistic fuzzy environments.

Using the parametric score function of Nayagam et al. [28], we have

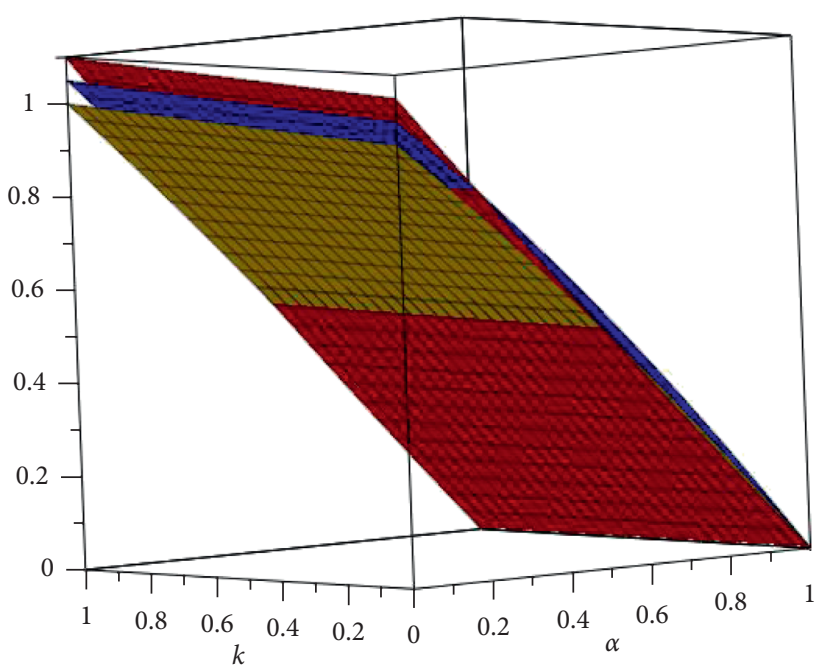

Figure 10: $S_{\alpha, k}$ index of three TraIFNs $\widetilde{A}, \widetilde{B}$, and $\widetilde{C}$.

$$
\begin{aligned}
& J(\widetilde{A}, \lambda)=\frac{0.6933(1-\lambda)+0.6817 \lambda}{1+0.4267 \lambda+0.4817(1-\lambda)}, \\
& J(\widetilde{B}, \lambda)=\frac{0.7633(1-\lambda)+0.7917 \lambda}{1+0.3633 \lambda+0.3917(1-\lambda)}, \\
& J(\widetilde{C}, \lambda)=\frac{0.7933(1-\lambda)+0.8200 \lambda}{1+0.3933 \lambda+0.4200(1-\lambda)} . \quad \forall \lambda \in[0,1] .
\end{aligned}
$$

We can see that, for all amounts of $\lambda$ parameter $(\lambda \in[0,1])$, we obtain $J(\widetilde{A})<J(\widetilde{B})<J(\widetilde{C})$ (see Figure 13), so from the work of Nayagam et al. [28], $\widetilde{A} \prec \widetilde{B} \prec \widetilde{C}, \forall \lambda \in[0,1]$. This result is the same as our obtained ranking results for $0.2<\alpha<0.5, k \in[0,1]$.

If we use Ye [15] index, we obtain $\operatorname{EV}(\widetilde{A})=0.4875$, $\operatorname{EV}(\widetilde{B})=0.5375$, and $\operatorname{EV}(\widetilde{C})=0.5125$, so we have $\widetilde{A} \prec \widetilde{C} \prec \widetilde{B}$, which agrees with our results for $0 \leq \alpha<0.2$ and $k \in[0,1]$.

In another paper, Lakshmana Gomathi Nayagam et al. [32] proposed a nonparametric index for ranking IFNs. By considering Lakshmana Gomathi Nayagam et al.'s [32] index, we obtain $L(\widetilde{A})=0.3087, L(\widetilde{B})=0.2987$, and $L(\widetilde{C})=0.2824$. Thus, based on their index, we have $\widetilde{C} \prec \widetilde{B} \prec \widetilde{A}$ which is not achieved by Ye [15], Chutia and Saikia [25], and our proposed method.

From the abovementioned examples, we can see that the proposed parametric method has appropriate discrimination power to rank IFNs based on the decision level $(\alpha)$ and hesitation degree $(k)$.

\section{Application in Intuitionistic Fuzzy Network DEA (IFNDEA) Problems}

In this section, to propose the advantages of the suggested index in application problems, we apply it to solve IFDEA and IFNDEA problems. 


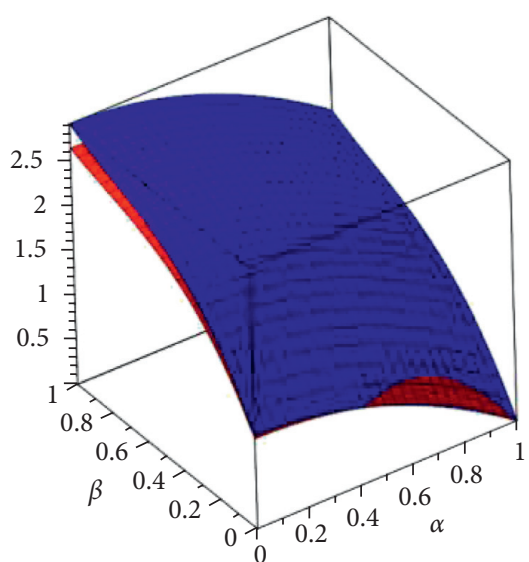

(a)

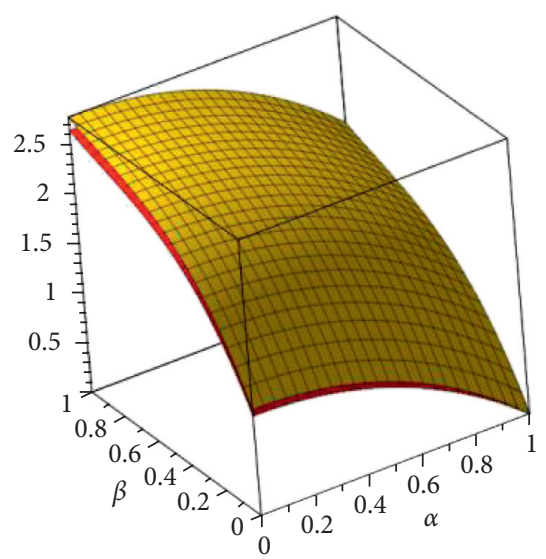

(b)

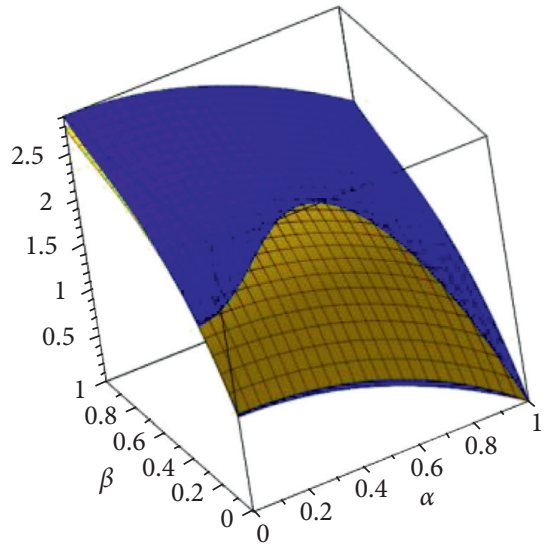

(c)

Figure 11: $V_{\alpha, \beta}$ functions of $\widetilde{A}$ and $\widetilde{B}, \widetilde{A}$ and $\widetilde{C}$, and $\widetilde{B}$ and $\widetilde{C}$ from Chutia and Saikia's [25] method.

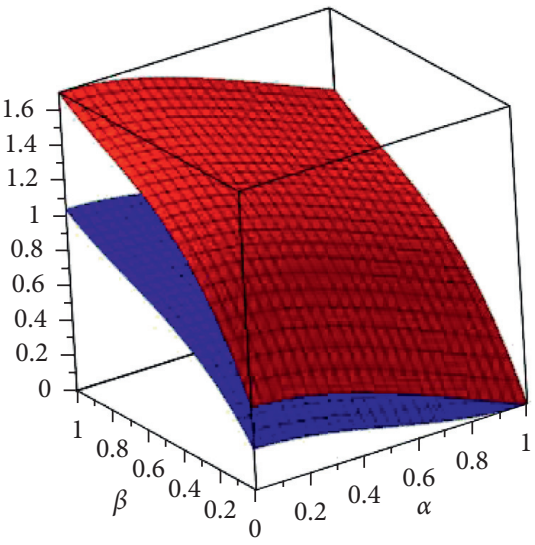

(a)

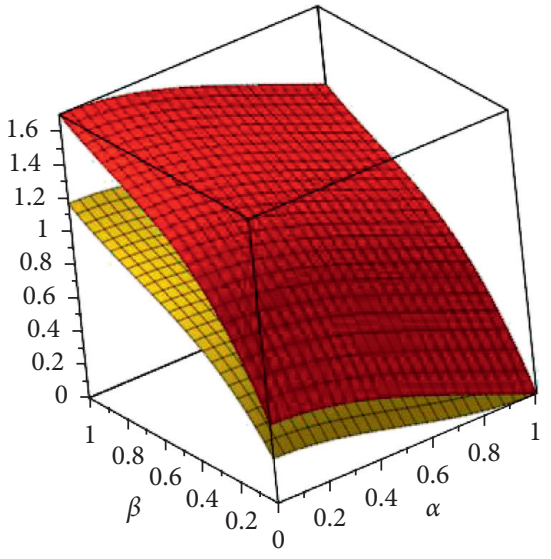

(b)

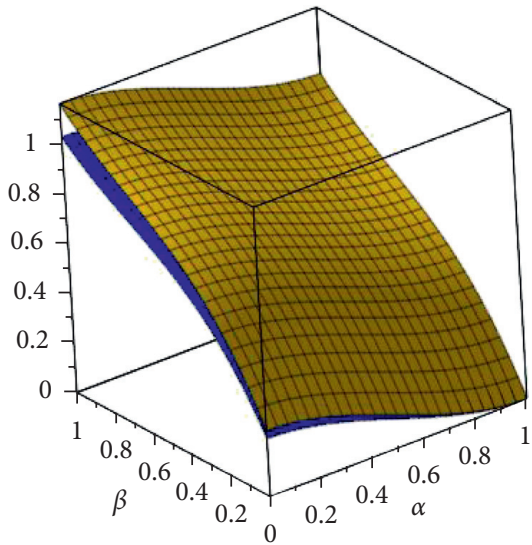

(c)

Figure 12: $A_{\alpha, \beta}$ functions of $\widetilde{A}$ and $\widetilde{B}, \widetilde{A}$ and $\widetilde{C}$, and $\widetilde{B}$ and $\widetilde{C}$ from Chutia and Saikia's [25] method.

4.1. Intuitionistic Fuzzy DEA (IFDEA) Problem. DEA is an optimization problem in a linear form to evaluate the efficiency of decision-making units (DMUs) with multipleinput/multiple-outputs assumptions. Despite the advantages of the traditional DEA models, there are some drawbacks in practical applications.

One drawback is that they do not work with noncrispy and uncertainty data in real-life problems. As we know, IFNs can efficiently describe fuzzy appearing in the data in the presence of hesitation. By considering IFNs in DEA problems, we should solve an IFDEA problem. Figure 14 shows an IFDEA model with $m$ IF inputs $\left(\widetilde{x}_{i j}, i=1, \ldots, m\right)$ and $s$ IF outputs $\left(\tilde{y}_{r j}, r=1, \ldots, s\right)$.

There have been suggested various DEA models for evaluating the efficiency of DMUs. Among them, the Charnes, Cooper, and Rhodes (CCR) model proposed by Charnes et al. [48] is the dominant DEA model. Considering IF inputs and IF outputs, the basic CCR model is converted to the following IFCCR model:

$$
\begin{aligned}
\tilde{\theta}_{o}= & \max \sum_{r=1}^{s} u_{r} \tilde{y}_{r o}, \\
\text { s.t. } & \sum_{i=1}^{m} v_{i} \tilde{x}_{i o}=1, \\
& \sum_{r=1}^{s} u_{r} \tilde{y}_{r j}-\sum_{i=1}^{m} v_{i} \tilde{x}_{i j} \leq 0, \quad j=1, \ldots, n, \\
& v_{i}, u_{r} \geq 0, i=1, \ldots, m, r=1, \ldots, s,
\end{aligned}
$$

where $\tilde{\theta}_{o}$ is the IF efficiency score of the DMU under evaluation (DMU ${ }_{o}$ ) and $v_{i}$ and $u_{r}$ are the given weights to IF input $i$ and IF output $r$, respectively.

By substituting the IF inputs and IF outputs with their assigned $S_{\alpha, k}$ functions, the abovementioned IFCCR model is transformed into the following parametric programming which is dependent on decision level $(\alpha)$ and hesitation degree $(k)$. 


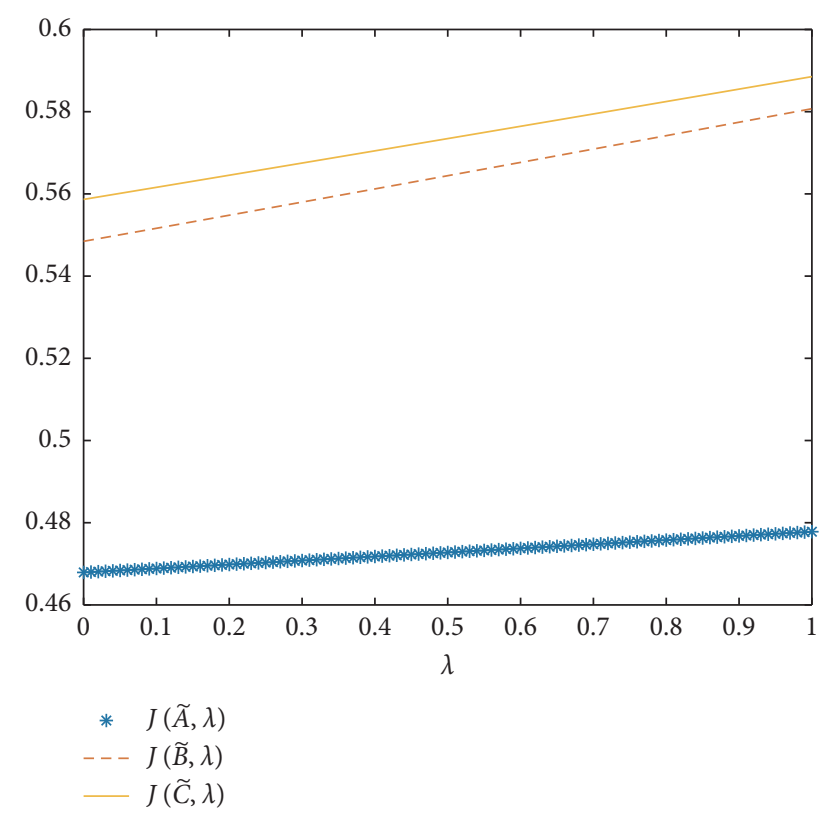

Figure 13: $J(\widetilde{A}, \lambda), J(\widetilde{B}, \lambda)$, and $J(\widetilde{C}, \lambda)$ score functions from Nayagam et al.'s [28] method.

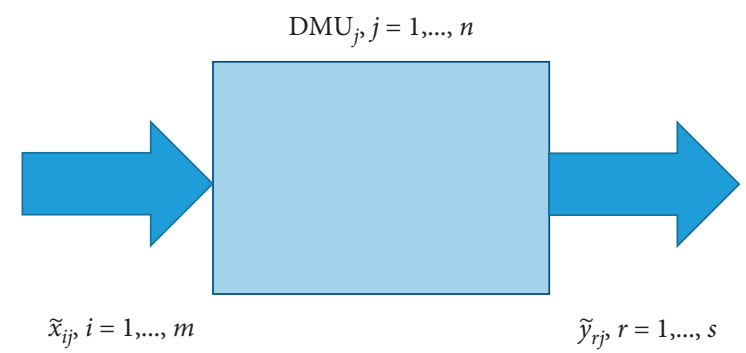

FIGURE 14: A DMU with $m$ IF inputs and $s$ IF outputs.

$$
\begin{aligned}
& \theta_{o}(\alpha, k)=\max \sum_{r=1}^{s} u_{r} S_{\alpha, k}\left(\tilde{y}_{r o}\right), \\
& \text { s.t. } \quad \sum_{i=1}^{m} v_{i} S_{\alpha, k}\left(\tilde{x}_{i o}\right)=1, \\
& \quad \sum_{r=1}^{s} u_{r} S_{\alpha, k}\left(\tilde{y}_{r j}\right)-\sum_{i=1}^{m} v_{i} S_{\alpha, k}\left(\tilde{x}_{i j}\right) \leq 0, \quad j=1, \ldots, n, \\
& \quad v_{i}, u_{r} \geq 0, i=1, \ldots, m, r=1, \ldots, s,
\end{aligned}
$$

where $\quad 0 \leq \alpha<w\left(w=\min \left\{w_{\tilde{x}_{i j}}, w_{\tilde{y}_{r j}} / i=1, \ldots, m, r=1\right.\right.$, $\ldots, s, j=1, \ldots, n\})$ and $0 \leq k \leq 1$.

Clearly, after determining the amounts of decision level $(\alpha)$ and hesitation degree $(k)$, the abovementioned parametric programming model will be converted to a simple linear program. Thus, we can easily compute the efficiency of DMUs for different amounts of $\alpha$ and $k$. Generally, the amounts of $\alpha$ and $k$ will be selected based on the decision maker's idea. If the decision maker considers $\alpha$ close to one, only the elements of an IFN with high membership values are of importance, and so, the obtained efficiency results are made with a high-level decision. Also, by assuming $\alpha$ close to zero, we obtain efficiency results under a low-level decision [53]. Moreover, if the decision maker considers $k$ close to zero, DMUs will be evaluated with a low hesitation degree (pessimistic decision), whereas for a high hesitation degree (optimistic decision), $k$ should be chosen near to one.

Although we display our method for the IFCCR model, it can be easily generalizable to other kinds of IFDEA models.

For more explanation, consider the following example that is proposed by Arya and Yadav [45].

Example 4. The following data are collected from 16 public hospitals of Meerut in the Indian state of Uttar Pradesh (Arya and Yadav [45]). There are two IF inputs and two IF outputs. The IF inputs are the total number of beds $\left(\tilde{x}_{1}\right)$ and the sum of pathologists and laboratory technicians $\left(\tilde{x}_{2}\right)$, and the IF outputs are the number of pathology operations $\left(\widetilde{y}_{1}\right)$ and the sum of plasters and tubal ligations $\left(\tilde{y}_{2}\right)$ (see Table 5).

Using model (30), the efficiency of 16 public hospitals for different amounts of decision level $(\alpha=0, \alpha=0.5$, and $\alpha=0.95)$ and hesitation degree $(k=0, k=0.5$, and $k=1)$ are calculated and shown in Table 6 . As can be seen, different efficiency and ranking results are obtained for different amounts of $\alpha$ and $k$. For example, for a fixed decision level $\alpha=0$, if we set $k=0$, then HCM will be efficient $(\theta=1)$ and ranked 1, but for $k=1$, HCM will be inefficient $(\theta=0.852528)$ and ranked 2. Also, for a fixed hesitation degree $k=0.5$, if we set $\alpha=0$, then the obtained efficiency result of GFD will be 0.517736 and ranked 8 , but for $\alpha=0.95$, the efficiency result of GFD is 0.743246 and ranked 3. Also, from Table 6 , for all the selected amounts of decision level $(\alpha)$ and hesitation degree $(k)$, the BD, BLD, and GBD hospitals are recognized as efficient hospitals and ranked one.

Inspired by the $\alpha$-level-based approach in fuzzy DEA (see Emrouznejad et al. [49]), Arya and Yadav [45] developed an approach to solve IFDEA models based on $\alpha$-cuts and $\beta$-cuts. The method provided lower and upper bounds of DMUs' efficiencies for different amounts of $\alpha$ and $\beta$. Then, by applying Chen and Klein's [50] index, they combined the obtained lower and upper bounds to rank DMUs. This approach assigns a wide range of efficiencies to a DMU for different amounts of $\alpha$ and $\beta$. Specifically, when they combine these obtained efficiency ranges, it can lead to incorrect ranking results. To illustrate, consider the obtained ranking results for 16 public hospitals based on Arya and Yadav's [45] method that is shown in the last column of Table 6.

Evidently, there are fundamental differences between our proposed method and Arya and Yadav's [45] method in ranking DMUs.

For example, if we compare inputs and outputs of GBD and MC hospitals, the outputs of GBD will be larger than the outputs of MC, and the inputs of GBD are smaller than the inputs of MC. Thus, the obtained efficiency and ranking results of GBD should be better than that of MC. Our method confirms this result for different amounts of $\alpha$ and 
TABle 5: IFNs as inputs and outputs of 16 public hospitals of Meerut in the Indian state of Uttar Pradesh (Arya and Yadav [45]).

\begin{tabular}{|c|c|c|c|c|}
\hline \multirow{2}{*}{ DMUs } & \multicolumn{2}{|l|}{ Inputs } & \multicolumn{2}{|r|}{ Outputs } \\
\hline & $\tilde{x}_{1}$ & $\tilde{x}_{2}$ & $\tilde{y}_{1}$ & $\tilde{y}_{2}$ \\
\hline $\mathrm{BC}$ & $(140,144,150 ; 135,144,155)$ & $(2,2,8 ; 1,2,15)$ & $\begin{array}{l}(83085,83089,83092 ; \\
83082,83089,83096)\end{array}$ & $(6,7,9 ; 5,7,12)$ \\
\hline $\mathrm{BD}$ & $(18,20,25 ; 15,20,28)$ & $(2,3,5 ; 1,3,10)$ & $\begin{array}{l}(14105,14107,14110 \\
14103,14107,14115)\end{array}$ & $(897,900,904 ; 895,900,908)$ \\
\hline BLC & $(442,446,449 ; 440,446,451)$ & $(2,3,6 ; 1,3,10)$ & $\begin{array}{l}(115855,115858,115862 \\
115852,115858,115865)\end{array}$ & $(1910,1913,1915 ; 1908,1913,1919)$ \\
\hline BLD & $(145,149,152 ; 140,149,155)$ & $(1,1,4 ; 1,1,8)$ & $\begin{array}{l}(165120,165121,165125 \\
165118,165121,165129)\end{array}$ & $(1560,1563,1567 ; 1558,1563,1570)$ \\
\hline BLFD & $(58,60,63 ; 55,60,65)$ & $(1,1,6 ; 1,1,10)$ & $\begin{array}{l}(34095,34099,340105 \\
34092,34099,340110)\end{array}$ & $(1620,1622,1626 ; 1617,1622,1630)$ \\
\hline BLK & $(65,68,70 ; 62,68,75)$ & $(1,1,6 ; 1,1,10)$ & $\begin{array}{l}(12185,12189,12195 \\
12180,12189,12199)\end{array}$ & $(1280,1283,1287 ; 1277,1283,1290)$ \\
\hline GC & $(122,124,126 ; 120,124,130)$ & $(6,7,10 ; 4,7,15)$ & $\begin{array}{l}(65290,65293,65298 \\
65286,65293,652102)\end{array}$ & $(215,218,222 ; 210,218,227)$ \\
\hline GD & $(162,166,168 ; 160,166,172)$ & $(3,5,8 ; 2,5,12)$ & $\begin{array}{l}(258750,258754,258758 \\
258745,258754,258765)\end{array}$ & $(423,426,428 ; 420,426,432)$ \\
\hline GFD & $(65,68,75 ; 62,68,80)$ & $(1,1,5 ; 1,1,10)$ & $\begin{array}{l}\text { (77852, 77856, 77859; } \\
\text { 77850, 77856, 77865) }\end{array}$ & $(1160,1164,1168 ; 1155,1164,1175)$ \\
\hline GS & $(98,100,105 ; 95,100,115)$ & $(2,2,6 ; 1,2,12)$ & $\begin{array}{l}(79720,79725,79729 ; \\
79718,79725,79735)\end{array}$ & $(910,913,916 ; 905,913,920)$ \\
\hline GBC & $(130,132,135 ; 125,132,145)$ & $(2,2,7 ; 1,2,13)$ & $\begin{array}{l}(25385,25387,25392 \\
25380,25387,25397)\end{array}$ & $(1756,1761,1765 ; 1750,1761,1770)$ \\
\hline GBD & $(97,100,105 ; 95,100,110)$ & $(2,3,8 ; 1,3,14)$ & $\begin{array}{l}(297445,297449,297453 \\
297440,297449,297458)\end{array}$ & $(4000,4004,4008 ; 3995,4004,4012)$ \\
\hline MC & $(248,250,255 ; 245,250,260)$ & $(6,8,12 ; 5,8,15)$ & $\begin{array}{l}(61190,61192,61196 \\
61185,61192,611102)\end{array}$ & $(635,638,642 ; 630,638,648)$ \\
\hline $\mathrm{MD}$ & $(248,250,255 ; 245,250,258)$ & $(6,8,13 ; 4,8,15)$ & $\begin{array}{l}(129432,129435,129438 \\
129430,129435,129445)\end{array}$ & $(1050,1052,1057 ; 1046,1052,1062)$ \\
\hline MFD & $(90,93,95 ; 85,93,100)$ & $(2,2,8 ; 1,2,13)$ & $\begin{array}{l}(78275,78278,78282 ; \\
78272,78278,78288)\end{array}$ & $(1600,1606,1610 ; 1595,1606,1610)$ \\
\hline $\mathrm{HCM}$ & $100,104,110 ; 95,104,120)$ & $(1,1,5 ; 1,1,10)$ & $\begin{array}{l}(44902,44906,44909 ; \\
44900,44906,44916)\end{array}$ & $(2110,2113,2116 ; 2106,2113,2120)$ \\
\hline
\end{tabular}

k. Despite that, MC is considered better than GBD by Arya and Yadav's [45] method (MC ranked 2 and GBD ranked $10)$.

Similarly, the outputs of GBD are larger than the outputs of GC, and the inputs of GBD are smaller than the inputs of GC. Hence, GBD should be more efficient than GC. Our method confirms this result for different amounts of $\alpha$ and $k$, but by Arya and Yadav's [45] method, GC is considered better than GBD (GC ranked 1 and GBD ranked 10).

4.2. Intuitionistic Fuzzy Network DEA (IFNDEA) Problems. Another drawback of traditional DEA models is that the structure of the decision-making units (DMUs) is assumed to be a black box, which takes no account of internal relations, operations, and structures. However, in real-world applications, we face DEA problems with important internal structures that cannot be ignored. Network DEA (NDEA) is an attempt to consider important interior structures of DMUs to DEA models. Assuming intuitionistic fuzzy data in an NDEA model, we will have an IFNDEA model. Among the various proposed structures, series and parallel are the two famous structures of NDEA models. But, although there are many papers on FDEA, IFDEA, and FNDEA fields, we found only one paper on IFNDEA (see [47]). In the work of Ameri et al. [47], IFNs are considered as inputs and outputs of a parallel NDEA model. In the following sections, we apply our proposed method in both series and parallel structures. Also, in a real-world application of the parallel IFNDEA models, the obtained results from our proposed method are compared with those of Ameri et al. [47].

4.2.1. Series Structure. Among all the proposed NDEA models, two-stage network processes have the simplest structure.

Consider a simple two-stage IFNDEA model in series form with $m$ IF inputs $\left(\tilde{x}_{i j}, i=1, \ldots, m\right), d$ IF intermediates $\left(\widetilde{z}_{d j}, d=1, \ldots, D\right)$, and $s$ IF outputs $\left(\widetilde{y}_{r j}, r=1, \ldots, s\right)$ data (Figure 15).

Different approaches have been proposed to evaluate the efficiency of DMUs in a two-stage NDEA model (see Cook and Zhu [54]), the multiplicative method initially proposed by Kao and Hwang [55] being well-known. In this method, overall efficiency is defined as a production of the stage's efficiencies. 


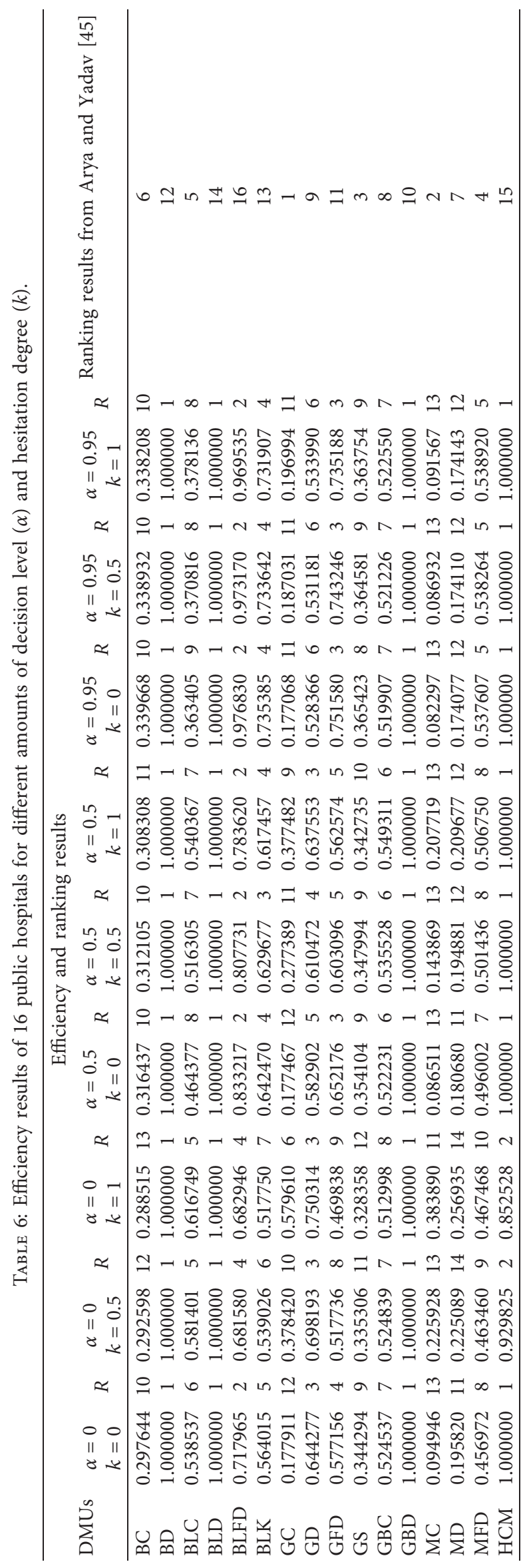




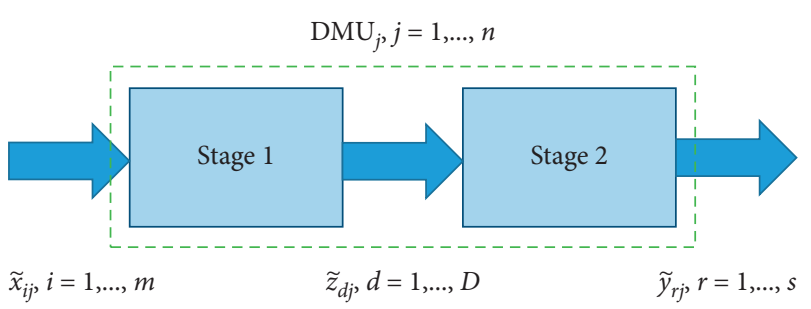

FIgURE 15: The generic IF two-stage network process.

If we consider IFNs for inputs, intermediates, and outputs data, the multiplicative two-stage IFNDEA model will be as follows:

$$
\begin{aligned}
\tilde{\theta}_{o}= & \max \sum_{r=1}^{s} u_{r} \tilde{y}_{r o}, \\
\text { s.t. } \quad & \sum_{i=1}^{m} v_{i} \tilde{x}_{i o}=1, \\
& \sum_{r=1}^{s} u_{r} \widetilde{y}_{r j}-\sum_{d=1}^{D} w_{d} \widetilde{z}_{d j} \leq 0, \quad j=1, \ldots, n, \\
& \sum_{d=1}^{D} w_{d} \widetilde{z}_{d j}-\sum_{i=1}^{m} v_{i} \tilde{x}_{i j} \leq 0, \quad j=1, \ldots, n, \\
& v_{i}, w_{d}, u_{r} \geq 0, i=1, \ldots, m, d=1, \ldots, D, r=1, \ldots, s,
\end{aligned}
$$

where $\tilde{\theta}_{o}$ is the IF efficiency score of the DMU under evaluation $\left(\mathrm{DMU}_{o}\right)$ and $v_{i}, w_{d}$, and $u_{r}$ are the given weights to IF input $i$, IF intermediate data $d$, and IF output $r$, respectively.

Now, by substituting the IF input, IF intermediate, and IF output data with their assigned $S_{\alpha, k}$ functions, the twostage IFNDEA model will be converted to the following parametric program:

$$
\begin{array}{ll}
\theta_{o}(\alpha, k)=\max & \sum_{r=1}^{s} u_{r} S_{\alpha, k}\left(\widetilde{y}_{r o}\right), \\
\text { s.t. } & \sum_{i=1}^{m} v_{i} S_{\alpha, k}\left(\widetilde{x}_{i o}\right)=1, \\
& \sum_{r=1}^{s} u_{r} S_{\alpha, k}\left(\widetilde{y}_{r j}\right)-\sum_{d=1}^{D} w_{d} S_{\alpha, k}\left(\widetilde{z}_{d j}\right) \leq 0, \quad j=1, \ldots, n, \\
& \sum_{d=1}^{D} w_{d} S_{\alpha, k}\left(\widetilde{z}_{d j}\right)-\sum_{i=1}^{m} v_{i} S_{\alpha, k}\left(\widetilde{x}_{i j}\right) \leq 0, \quad j=1, \ldots n, \\
& v_{i}, w_{d}, u_{r} \geq 0, i=1, \ldots, m, d=1, \ldots, D, r=1, \ldots, s,
\end{array}
$$

where $0 \leq \alpha<w\left(w=\min \left\{w_{\tilde{x}_{i j}}, w_{\tilde{z}_{d r j}}, w_{\tilde{y}_{r j}} / i=1, \ldots, m, d=\right.\right.$ $1, \ldots, D, r=1, \ldots, s, j=1, \ldots, n\})$ and $0 \leq k \leq 1$.

Based on the decision maker's opinion, by appropriate selection of the decision level $(\alpha)$ and hesitation degree $(k)$, model (32) will be converted to a linear program.

The following numerical example is proposed to illustrate the abovementioned method.
Example 5. Consider five hypothetical DMUs with two IF inputs, two IF intermediate products, and two IF outputs in a two-stage NDEA structure (Table 7).

As can be seen from Table 7, all the input, intermediate, and output data are assumed to be asymmetric TraIFNs. Expectedly, different selection of the decision level (alpha) and hesitation degree $(k)$ led to different ranking and efficiency results. Using model (32), the efficiency results of DMUs are calculated and shown in Table 8 for different amounts of decision level ( $\alpha=0, \alpha=0.5$, and $\alpha=0.75)$ and hesitation degree $(k=0, k=0.5$, and $k=1)$. We can observe that different amounts of $\alpha$ and $k$ have led to different efficiency and ranking results. For example, the efficiency score of DMU1 for $\alpha=0, k=1$ (a low-level decision with a high hesitation degree) is 0.377979 and ranked 2, but for $\alpha=0.75, k=0$ (a high-level decision with a low hesitation degree), the score is 0.114912 and it ranked 5. Also, the efficiency score of DMU4 for $\alpha=0.75, k=0$ (a high-level decision with a low hesitation degree) is 0.498449 and ranked 1 , but for $\alpha=0.75, k=1$ (a high-level decision with a high hesitation degree), the score is 0.223572 and it ranked 4 . In most cases, DMU2 is the worst DMU and DMU3 is the best DMU. Also, DMU4 and DMU5 experience most changes in their rankings (from the first to fourth).

Since crisp or fuzzy numbers are special cases of IFNs, our proposed method can solve NDEA models with a collection of crisp, fuzzy, and intuitionistic fuzzy numbers as data too.

4.2.2. Parallel Structure. Unlike series structures, there is no flow of intermediate products in the parallel structures. In parallel structures, the input and output data are divided among processes (Figure 16).

Consider a parallel IFNDEA model with $q$ independent processes (Figure 16). In this model, each process $p$ uses IF inputs $\widetilde{x}_{i}^{(p)}, i \in I^{(p)}$ to produce IF outputs $\widetilde{y}_{r}^{(p)}, r \in O^{(p)}$. In this system, the sum of the IF inputs used by all the $q$ processes $\left(\sum_{p=1}^{q} \tilde{x}_{i j}^{(p)}=\tilde{x}_{i j}\right)$ is equal to the system IF inputs, and the sum of the IF outputs used by all the $q$ processes $\left(\sum_{p=1}^{q} \tilde{y}_{r j}^{(p)}=\tilde{y}_{r j}\right)$ is equal to the system IF outputs. Based on Kao's [56] method, to evaluate the system and process efficiencies, first, the following IFNDEA model should be solved:

$$
\begin{aligned}
\tilde{\theta}_{o}= & \max \sum_{r=1}^{s} u_{r} \tilde{y}_{r o}, \\
\text { s.t. } & \sum_{i=1}^{m} v_{i} \tilde{x}_{i o}=1, \\
& \sum_{r \in O^{(p)}} u_{r} \tilde{y}_{r j}^{(p)}-\sum_{i \in I^{(p)}} v_{i} \tilde{x}_{i j}^{(p)} \leq 0, \quad p=1, \ldots, q, j=1, \ldots, n, \\
& v_{i}, u_{r} \geq 0, i=1, \ldots, m, r=1, \ldots, s .
\end{aligned}
$$

By our proposed method, we should first substitute the IF inputs and IF outputs with their assigned $S_{\alpha, k}$ functions. So, the abovementioned IFNDEA model will transform into 


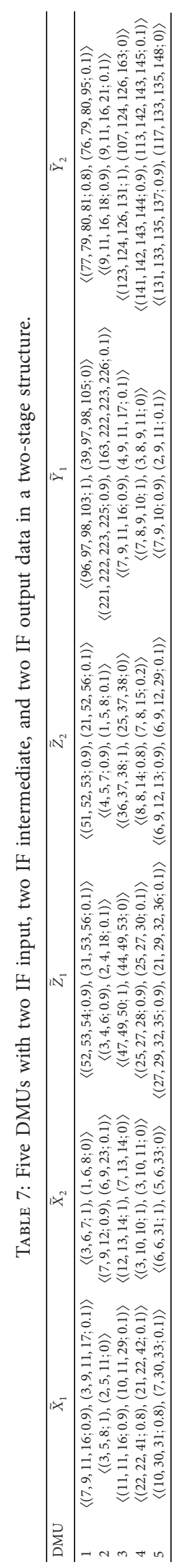




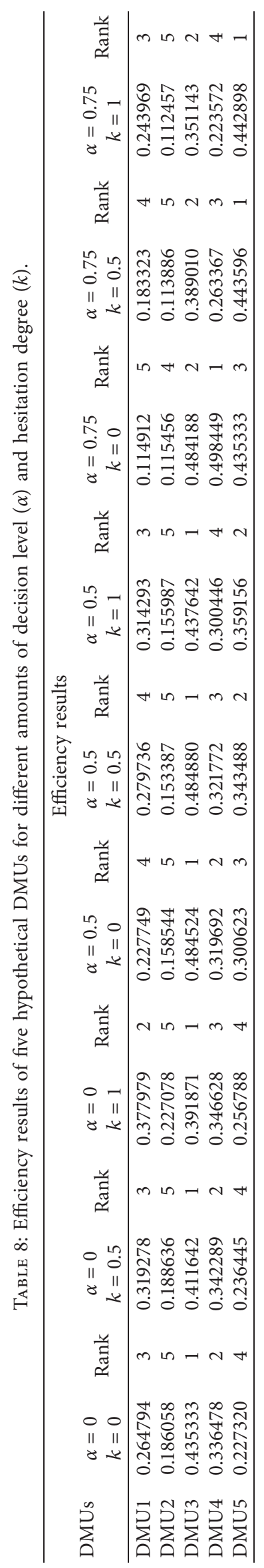




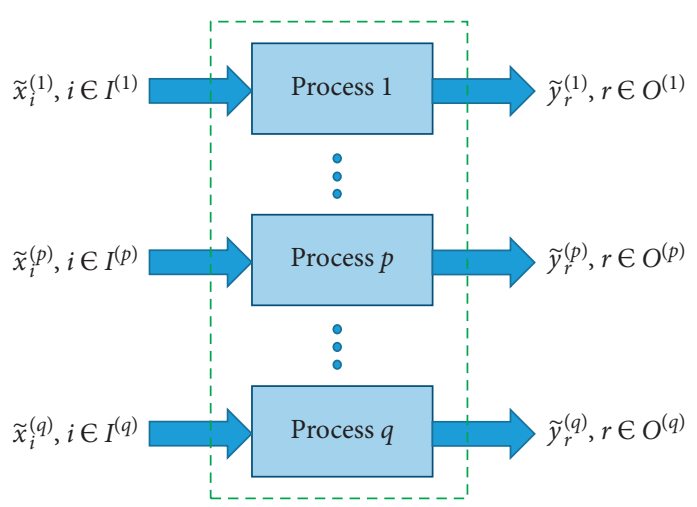

FIgURe 16: A parallel system.

the following parametric programming which is dependent on decision level $(\alpha)$ and hesitation degree $(k)$ :

$$
\begin{aligned}
& \theta_{o}(\alpha, k)=\max \sum_{r=1}^{s} u_{r} S_{\alpha, k}\left(\tilde{y}_{r o}\right), \\
& \text { s.t. } \quad \sum_{i=1}^{m} v_{i} \tilde{x}_{i o}=1, \\
& \sum_{r \in O^{(p)}} u_{r} S_{\alpha, k}\left(\tilde{y}_{r j}^{(p)}\right)-\sum_{i \in I^{(p)}} v_{i} S_{\alpha, k}\left(\tilde{x}_{i j}^{(p)}\right) \leq 0, \\
& \quad p=1, \ldots, q, j=1, \ldots, n, \\
& v_{i}, u_{r} \geq 0, i=1, \ldots, m, r=1, \ldots, s,
\end{aligned}
$$

where $\quad 0 \leq \alpha<w\left(w=\min \left\{w_{\tilde{x}_{i j}}, w_{\tilde{y}_{r j}} / i=1, \ldots, m, r=1\right.\right.$, $\ldots, s, j=1, \ldots, n\})$ and $0 \leq k \leq 1$.

Consequently, after the selection of the decision level $(\alpha)$ and hesitation degree $(k)$, we can solve the obtained linear program.

Let $\left(v_{i}^{*}, u_{r}^{*}\right)$ be an optimal solution of the model (34). We can calculate the process and system efficiencies by the following formulas:

$$
\begin{gathered}
\text { Process efficiencies : } \theta_{o}^{(p)}=\frac{\sum_{r \in O^{(p)}} u_{r}^{*} S_{\alpha, k}\left(\tilde{y}_{r o}^{(p)}\right)}{\sum_{i \in I^{(p)}} v_{i}^{*} S_{\alpha, k}\left(\tilde{x}_{i o}^{(p)}\right)}, \quad p=1, \ldots, q, \\
\text { System efficiencies : } \theta_{o}=\sum_{r=1}^{s} u_{r}^{*} S_{\alpha, k}\left(\widetilde{y}_{r o}\right) .
\end{gathered}
$$

To apply our proposed method in a real-world application, we consider the following example proposed by Ameri et al. [47]. In Ameri et al.'s [47] method, by substituting the IF inputs and IF outputs with their assigned expected values (Grzegorzewski [13]), the model (34) is transformed into a linear program. Unlike our proposed method, in the work of Ameri [47], we are allowed only to use a specific group of IFNs (normal TraIFNs), and the decision maker's opinion is not considered in the decision-making process.

Example 6. Imam Khomeini is one of the most famous hospitals in Iran. For the purpose of self-assessment of the
Imam Khomeini hospital, Ameri et al. [47] collected the hospital data in eight different periods. So, we have eight DMUs that are made by one hospital data in these different eight periods of time. The internal structure of the hospital department is considered as a parallel structure with four processes in the following sections: internal, general surgery, pediatric, and CCU. Table 9 shows the hospital data, and we can see that each process has three inputs and four outputs. The input data are the physician $x_{1}$, the number of nurses $x_{2}$, and the number of beds $x_{3}$. Also, the output data are the bed occupancy percentage (BOP) $y_{1}$, satisfaction with physician performance $y_{2}$, satisfaction with nurse performance $y_{3}$, and satisfaction with environmental conditions $y_{4}$. As evident in Table $9, x_{1}, x_{2}, x_{3}$, and $y_{1}$ data are real numbers, while to control the uncertainty and for better modeling of the real data represented by organizations' decision makers, $y_{2}, y_{3}$, and $y_{4}$ data are represented by normal TriIFNs.

Using model 34, the efficiency results of the hospital and its sections are calculated for eight different periods of time (see Table 10). Unlike the previous example, in this example, there are no significant differences among the obtained efficiency results for the sections based on different values of the decision level $(\alpha)$ or hesitation degree $(k)$. To explain, all the input data and an input datum are real numbers. Also, the other three output data are not represented by asymmetric or intersected TriIFNs. From Table 10, the greatest differences among the obtained efficiency results for different amounts of $\alpha$ and $k$ is for the CCU section in part 6 (from $0.84959443 \quad(\alpha=0, k=1) \quad$ to 0.91640810 $(\alpha=0.95, k=0))$. We can see that there are not any efficient sections in parts $1,2,3$, and 5 . Also, the pediatrics section in parts 4 and 6, CCU section in parts 4 and 7, and general surgery section in part 8 are reported as efficient sections.

The obtained efficiency results from Ameri et al.'s [47] method is shown in the last column of Table 10. As can be seen, although the problem is solved with two different approaches, the obtained efficiency results from our proposed method are nearly identical to those of Ameri et al. [47]. However, the general surgery section in part 6 from our proposed method is an inefficient section for all the selected amounts of $\alpha$ and $k$, but from Ameri et al.'s [47] method, it is an efficient section. 
TABLE 9: Input and output of Imam Khomeini hospital ([47]).

\begin{tabular}{|c|c|c|c|c|c|c|c|}
\hline \multirow{2}{*}{ Periods } & \multicolumn{3}{|c|}{ Input } & \multicolumn{4}{|c|}{ Output } \\
\hline & $x_{1}$ & $x_{2}$ & $x_{3}$ & $y_{1}$ & $y_{2}$ & $y_{3}$ & $y_{4}$ \\
\hline Spring 2015 (part 1) & 33 & 70 & 103 & 15.5 & 63; 124, & 8,$295 ; 220$ & $(70,79,93 ; 49,79,108)$ \\
\hline al section & 12 & 24 & 47 & & 3,$47 ; 37$, & $(65,68,77$; & $(48,50,59 ; 32$ \\
\hline General surgery section & 10 & 15 & 24 & 32.22 & $(26,30,33 ; 24,30,35)$ & $(61,68,72 ; 58,68,74)$ & $(8,10,12 ; 6,10,13)$ \\
\hline Pediatrics section & 7 & 17 & 12 & 43.63 & $22,28,31 ; 20,28,33)$ & $(42,51,58 ; 37,51,61)$ & $(9,11,13 ; 7,11,15)$ \\
\hline CCU section & 4 & 14 & 20 & 75.20 & $15,48,52 ; 43,48,53)$ & $(72,81,88 ; 67,81,91)$ & $(5,8,9 ; 4,8,13)$ \\
\hline Summer 2015 & 37 & 71 & 108 & 212.74 & $(131,156,169 ; 119,156,199)$ & $(136,157,187 ; 116,157,217)$ & $(126,141,151 ; 114,141,162$ \\
\hline Internal section & 14 & 24 & 50 & & & $(35,40,42 ; 22,40,59)$ & \\
\hline General surgery section & 10 & 16 & 24 & 36.23 & $, 20,10,1$ & $(40,47,65 ; 38$, & , \\
\hline Pediatrics section & 8 & 16 & 13 & 38.58 & 140 & $(43,50,57 ; 41$ & $24 ; 17$ \\
\hline CCU section & 5 & 15 & 21 & 78.92 & $(28,30,33 ; 25,30,35)$ & $(18,20,23 ; 15,20$, & $(55,60,63 ; 52,60,64)$ \\
\hline Autuı & 40 & 70 & 114 & 203.57 & $(129,154,167 ; 1$ & $(209,224,256 ; 199$ & $(71,8$ \\
\hline Internal section & 15 & 26 & 50 & 79.23 & 36 & $(145,149,152 ; 14$ & $(26$ \\
\hline Gene & 12 & 15 & 26 & & & & \\
\hline Pedi & 8 & 16 & 13 & 53.02 & & & \\
\hline & 5 & 13 & 25 & 45.36 & 32 & $(18,20,25$ & 22 \\
\hline Wir & 40 & 71 & 118 & 89 & 212) & $(210,225,242$ & \\
\hline & 13 & 25 & 52 & & & & \\
\hline ion & 14 & 16 & 27 & & & & \\
\hline Ped & 9 & 18 & 13 & 6 & $0)$ & 16) & \\
\hline & 4 & 12 & 26 & & & 7 & 2) \\
\hline & 44 & 73 & 121 & & 66) & & \\
\hline & 15 & 27 & 51 & & & $(25$ & \\
\hline & 15 & 15 & 29 & & & & 05) \\
\hline Ped & 10 & 18 & 16 & & & & \\
\hline $\mathrm{CCU}$ & 4 & 13 & 25 & 74.32 & & $(75,80,83 ;$ & 23 \\
\hline Sur & 42 & 82 & 126 & 239.84 & 254) & $(513,524,533 ;$ & $(233$ \\
\hline Inte & 12 & 30 & 55 & 52.56 & $2,32,36 ; 18,3$ & $(178,180,182 ; 176,180,184)$ & \\
\hline & 15 & 16 & 30 & & & & \\
\hline Pec & 9 & 21 & 17 & & & 251) & \\
\hline $\mathrm{CO}$ & 6 & 15 & 24 & 8 & (1) & 6) & 2) \\
\hline $\mathrm{Au}$ & 50 & 90 & 136 & 258.8 & $(258,283,294 ; 240,283,301)$ & $(227,240,253 ; 217,240,265)$ & $(61,72,78 ; 51,72,87)$ \\
\hline & 15 & 32 & 53 & 65.14 & $(59,64,66 ; 46,64,68)$ & $(24,29,31 ; 22,29,35)$ & $(16,19,20 ; 14,19,22)$ \\
\hline Ger & 17 & 19 & 35 & & & $(128,129,130 ; 1$ & 13) \\
\hline Pediat & 10 & 21 & 22 & 35.24 & & & $(19,22,24 ; 1$ \\
\hline $\mathrm{CCU}$ & 8 & 18 & 26 & 74.31 & $(119,121,124 ; 118,121,125)$ & $(55,60,68 ; 52,60,74)$ & $(17,20,22 ; 14,20,25)$ \\
\hline & 54 & 96 & 140 & 228.34 & $(194,221,233 ; 181,221,248)$ & $(308,331,342 ; 298,331,351)$ & $(147,158,165 ; 139,158,173$ \\
\hline Int & 14 & 36 & 54 & & (10 2? 25.16 22 20) & $(17,20,25 ; 13,20,32)$ & $(12,14,16 ; 9,14,19)$ \\
\hline surgery sec & 20 & 23 & 38 & 48.25 & & $(74,86,89 ; 71,86,89)$ & $(115,118,119 ; 113,118,1$ \\
\hline cs section & 12 & 20 & 21 & 54.68 & $(31,32,34 ; 27,32 ; 36)$ & $(8,12,13 ; 7,12,14)$ & $(3,6,8 ; 2,6,9)$ \\
\hline CCU section & 8 & 17 & 27 & 68.56 & $(65,79,84 ; 62,79 ; 88)$ & $(209,213,215 ; 207,213,216)$ & $(17,20,22 ; 15,20,23)$ \\
\hline
\end{tabular}

As mentioned earlier, in our proposed method, the decision maker's idea is accounted for in the decisionmaking process by proper selection of the decision level (alpha cut) and the hesitation degree, and so, less information is lost in IFNs. But, in Ameri et al. [47], only a real number (expected value) is assigned to each IF data, and the decision maker does not play any role in the decisionmaking process.

Although we adopted our method in the multiplicative two-stage IFNDEA and parallel IFNDEA models, it will be easily generalizable to other kinds of IFNDEA models.

We can summarize the algorithm of the proposed method to solve IFNDEA models in real-world applications as follows:

Step 1. An appropriate IFNDEA model is chosen based on the structure of the real-world problem
Step 2. The input, intermediate, and output data of the problem are determined and represented by crisp, fuzzy, or intuitionistic fuzzy numbers

Step 3. The appropriate decision level $(\alpha)$ and hesitation degree $(k)$ are selected based on the decision maker's opinion

Step 4. Input, intermediate, and output data are replaced with their assigned $S_{\alpha, k}$ index

Step 5. The transformed IFNDEA model is solved to obtain the efficiency score of the DMU under evaluation based on the selected decision level $(\alpha)$ and hesitation degree $(k)$

As evident in the algorithm, after replacing input, intermediate, and output data with their assigned $S_{\alpha, k}$ indexes, we should solve a transformed IFNDEA model 
Table 10: Part and section efficiencies of Imam Khomeini hospital.

\begin{tabular}{|c|c|c|c|c|c|c|}
\hline \multicolumn{7}{|c|}{ Efficiency results } \\
\hline Periods & $\begin{array}{l}\alpha=0 \\
k=0\end{array}$ & $\begin{array}{l}\alpha=0 \\
k=1\end{array}$ & $\begin{array}{r}\alpha=0.5 \\
k=0.5\end{array}$ & $\begin{array}{c}\alpha=0.95 \\
k=0\end{array}$ & $\begin{array}{c}\alpha=0.95 \\
k=1\end{array}$ & Efficiency results from [47] \\
\hline Spring 2015 (part 1) & 0.52019383 & 0.52038297 & 0.52046751 & 0.52062196 & 0.52063228 & 053 \\
\hline Internal section & 0.37049740 & 0.37059818 & 0.37061554 & 0.37067324 & 0.37067916 & 0.37 \\
\hline General surgery section & 0.35814522 & 0.35863255 & 0.35849474 & 0.35857985 & 0.35860436 & 0.36 \\
\hline Pediatrics section & 0.61563699 & 0.61541665 & 0.61610918 & 0.61662677 & 0.61661733 & 0.62 \\
\hline CCU section & 0.93076892 & 0.93095326 & 0.93098360 & 0.93108750 & 0.93109774 & 0.93 \\
\hline Summer 2015 (part 2) & 0.52951119 & 0.53988654 & 0.53565751 & 0.53654424 & 0.53703037 & 0.58 \\
\hline Internal section & 0.40743351 & 0.44357556 & 0.42080410 & 0.41714803 & 0.41876904 & 0.63 \\
\hline General surgery section & 0.42609976 & 0.42637242 & 0.42719074 & 0.42799112 & 0.42801671 & 0.44 \\
\hline Pediatrics section & 0.50641785 & 0.49147257 & 0.50597856 & 0.51183970 & 0.51120391 & 0.42 \\
\hline CCU section & 0.92605580 & 0.91039378 & 0.92655494 & 0.93342257 & 0.93277853 & 0.78 \\
\hline Autumn 2015 (part 3) & 0.48110817 & 0.48354484 & 0.48412580 & 0.48568293 & 0.48580386 & 0.50 \\
\hline Internal section & 0.40205601 & 0.40546786 & 0.40464178 & 0.40534301 & 0.40551456 & 0.40 \\
\hline General surgery section & 0.37247189 & 0.37131625 & 0.37493114 & 0.37770132 & 0.37764297 & 0.46 \\
\hline Pediatrics section & 0.75733115 & 0.75863490 & 0.75845180 & 0.75884116 & 0.75890479 & 0.76 \\
\hline CCU section & 0.51040530 & 0.51621843 & 0.51629130 & 0.51882412 & 0.51911125 & 0.54 \\
\hline Winter 2016 (part 4) & 0.63380952 & 0.64802176 & 0.63747987 & 0.63465362 & 0.63527938 & 0.66 \\
\hline Internal section & 0.21659433 & 0.21433258 & 0.21818399 & 0.22055849 & 0.22047566 & 0.22 \\
\hline General surgery section & 0.83185347 & 0.89473301 & 0.84257872 & 0.82528746 & 0.82800716 & 0.91 \\
\hline Pediatrics section & 1.00000000 & 1.00000000 & 1.00000000 & 1.00000000 & 1.00000000 & 1 \\
\hline CCU section & 1.00000000 & 1.00000000 & 1.00000000 & 1.00000000 & 1.00000000 & 1 \\
\hline Spring 2016 (part 5) & 0.76329145 & 0.75832779 & 0.76260960 & 0.76438792 & 0.76415083 & 0.80 \\
\hline Internal section & 0.70277356 & 0.69897033 & 0.70227099 & 0.70380962 & 0.70363143 & 0.75 \\
\hline General surgery section & 0.93057681 & 0.91137860 & 0.92792953 & 0.93441729 & 0.93347599 & 0.97 \\
\hline Pediatrics section & 0.64641599 & 0.64407482 & 0.63887149 & 0.63329659 & 0.63319397 & 0.67 \\
\hline CCU section & 0.81223795 & 0.82087539 & 0.82326123 & 0.82925228 & 0.82969195 & 0.82 \\
\hline Summer 2016 (part 6) & 0.75332545 & 0.74449545 & 0.75154348 & 0.75410919 & 0.75366959 & 0.77 \\
\hline Internal section & 0.47306006 & 0.47362012 & 0.47597925 & 0.47833996 & 0.47837178 & 0.48 \\
\hline General surgery section & 0.94603193 & 0.95661246 & 0.94246705 & 0.93446320 & 0.93495669 & 1 \\
\hline Pediatrics section & 1.00000000 & 1.00000000 & 1.00000000 & 1.00000000 & 1.00000000 & 1 \\
\hline CCU section & 0.91055221 & 0.84959443 & 0.89842999 & 0.91640810 & 0.91336049 & 0.93 \\
\hline Autumn 2016 (part 7) & 0.56422335 & 0.56300855 & 0.56602734 & 0.56823501 & 0.56817147 & 0.57 \\
\hline Internal section & 0.36825787 & 0.36288963 & 0.36829979 & 0.37090507 & 0.37063315 & 0.37 \\
\hline General surgery section & 0.62017078 & 0.62402281 & 0.62582484 & 0.62908574 & 0.62927534 & 0.63 \\
\hline Pediatrics section & 0.43663412 & 0.43604903 & 0.43903647 & 0.44147849 & 0.44144522 & 0.44 \\
\hline CCU section & 1.00000000 & 1.00000000 & 1.00000000 & 1.00000000 & 1.00000000 & 1 \\
\hline Winter 2017 (part 8) & 0.49968659 & 0.50521119 & 0.50456206 & 0.50641263 & 0.50666320 & 0.52 \\
\hline Internal section & 0.13315405 & 0.14940829 & 0.13900997 & 0.13680373 & 0.13757147 & 0.16 \\
\hline General surgery section & 1.00000000 & 1.00000000 & 1.00000000 & 1.00000000 & 1.00000000 & 1 \\
\hline Pediatrics section & 0.29673692 & 0.33097934 & 0.30789228 & 0.30290102 & 0.30442367 & 0.34 \\
\hline CCU section & 0.75839956 & 0.71122734 & 0.76033076 & 0.78346830 & 0.78121672 & 0.76 \\
\hline
\end{tabular}

that is a linear-programming model. Different methods are proposed for solving linear-programming problems such as the simplex method or Karmarkar method. Thus, the time complexity of our proposed method depends on the selected method for solving the obtained linear program.

\section{Conclusions}

IFNs can efficiently model noncrispy and uncertainty in real-world data. Ranking is the second most important issue after IFNs definition. In this study, a new parametric method was proposed for ranking IFNs, and by appropriate examples, its superiority over some other methods was explained. Engaging the decision maker in the decisionmaking process is an important advantage of the proposed method. It was observed that the decision maker's opinion about the decision level and hesitation degree parameters can affect the obtained ranking results. Many proposed methods to date rank only a special group of IFNs (e.g., TriIFNs, TraIFNs, or normal IFNs), but the proposed method is able to simultaneously rank all kinds of IFNs. Also, to show the ability of the proposed method in realworld applications, we applied our method to solve IFDEA, two-stage IFNDEA, and parallel IFNDEA models. The proposed approach is easily generalizable to solve other kinds of IFNDEA models too.

As future studies, we aim to apply our proposed method to multicriteria decision-making (MCDM) techniques, such as the analytic hierarchy process (AHP), best-worst method (BWM), and VIKOR, to solve intuitionistic fuzzy MCDM problems. 


\section{Data Availability}

The data used to support the findings of this study are available from the corresponding author upon request.

\section{Conflicts of Interest}

The authors declare that there are no conflicts of interest in this paper.

\section{References}

[1] K. T. Atanassov, "Intuitionistic fuzzy sets," Fuzzy Sets and Systems, vol. 20, no. 1, pp. 87-96, 1986.

[2] K. T. Atanassov, "Intuitionistic fuzzy sets: theory and applications," Studies in Fuzziness and Soft Computing, Springer, Berlin, Germany, 1999.

[3] L. A. Zadeh, "Fuzzy sets," Information and Control, vol. 8, no. 3, pp. 338-353, 1965.

[4] L. A. Zadeh, "Similarity relations and fuzzy orderings," Information Sciences, vol. 3, no. 2, pp. 177-200, 1971.

[5] D.-F. Li, "Decision and game theory in management with intuitionistic fuzzy sets," Studies in Fuzziness and Soft Computing, Springer, vol. 308, Berlin, Germany, 2014.

[6] P. Liu, S.-M. Chen, and Y. Wang, "Multiattribute group decision making based on intuitionistic fuzzy partitioned Maclaurin symmetric mean operators," Information Sciences, vol. 512, pp. 830-854, 2020.

[7] M. Sarwar and M. Akram, "An algorithm for computing certain metrics in intuitionistic fuzzy graphs," Journal of Intelligent \& Fuzzy Systems, vol. 30, no. 4, pp. 2405-2416, 2016.

[8] E. Szmidt, "Distances and similarities in intuitionistic fuzzy sets," Studies in Fuzziness and Soft Computing, Springer, vol. 307, Cham, Switzerland, 2014.

[9] M. Akram, M. Sarwar, and R. A. Borzooei, "A novel decisionmaking approach based on hypergraphs in intuitionistic fuzzy environment," Journal of Intelligent \& Fuzzy Systems, vol. 35, no. 2, pp. 1905-1922, 2018.

[10] P. Burillo, H. Bustince, and V. Mohedano, Some Definition of Intuitionistic Fuzzy Number, Fuzzy Based Expert Systems, Fuzzy Bulgarian Enthusiasts, Sofia, Bulgaria, 1994.

[11] P. Liu and P. Wang, "Multiple attribute group decision making method based on intuitionistic fuzzy einstein interactive operations," International Journal of Fuzzy Systems, vol. 22, no. 3, pp. 790-809, 2020.

[12] P. Wang and P. Liu, "Some maclaurin symmetric mean aggregation operators based on schweizer-sklar operations for intuitionistic fuzzy numbers and their application to decision making," Journal of Intelligent \& Fuzzy Systems, vol. 36, no. 4, pp. 3801-3824, 2019.

[13] P. Grzegorzewski, "Distances and orderings in a family of intuitionistic fuzzy numbers," in Proceedings of the EUSFLAT Conference, pp. 223-227, Zittau, Germany, September 2003.

[14] P. Grzegorzewski, "Metrics and orders in space of fuzzy numbers," Fuzzy Sets and Systems, vol. 97, no. 1, pp. 83-94, 1998.

[15] J. Ye, "Expected value method for intuitionistic trapezoidal fuzzy multicriteria decision-making problems," Expert Systems with Applications, vol. 38, no. 9, pp. 11730-11734, 2011.

[16] H. B. Mitchell, "Ranking-intuitionistic fuzzy numbers," International Journal of Uncertainty, Fuzziness and KnowledgeBased Systems, vol. 12, no. 3, pp. 377-386, 2004.
[17] W. Jianqiang and Z. Zhong, "Aggregation operators on intuitionistic trapezoidal fuzzy number and its application to multi-criteria decision making problems," Journal of Systems Engineering and Electronics, vol. 20, no. 2, pp. 321-326, 2009.

[18] J. Wang and Z. Zhang, "Multi-criteria decision-making method with incomplete certain information based on intuitionistic fuzzy number," Control and Decision, vol. 24, no. 2, pp. 226-230, 2009.

[19] H. M. Nehi, "A new ranking method for intuitionistic fuzzy numbers," International Journal of Fuzzy Systems, vol. 12, no. 1, pp. 80-86, 2010.

[20] K. P. Chiao, "Characteristic value of fuzzy number defined with parameter integral form," in Proceedings of the The Nineth National Conference on Fuzzy Theory and its Applications (Fuzzy 2001), Varanasi, India, November 2001.

[21] J.-X. Nan, D.-F. Li, and M.-J. Zhang, "A lexicographic method for matrix games with payoffs of triangular intuitionistic fuzzy numbers," International Journal of Computational Intelligence Systems, vol. 3, no. 3, pp. 280-289, 2010.

[22] T. Verma and A. Kumar, "A note on "a lexicographic method for matrix games with payoffs of triangular intuitionistic fuzzy numbers"' International Journal of Computational Intelligence Systems, vol. 8, no. 4, pp. 690-700, 2015.

[23] M. Delgado, M. A. Vila, and W. Voxman, "On a canonical representation of fuzzy numbers," Fuzzy Sets and Systems, vol. 93, no. 1, pp. 125-135, 1998.

[24] R. Chutia and B. Chutia, "A new method of ranking parametric form of fuzzy numbers using value and ambiguity," Applied Soft Computing, vol. 52, pp. 1154-1168, 2017.

[25] R. Chutia and S. Saikia, "Ranking intuitionistic fuzzy numbers at levels of decision-making and its application," Expert Systems, vol. 36, no. 2, 2018.

[26] D.-F. Li, "A ratio ranking method of triangular intuitionistic fuzzy numbers and its application to MADM problems," Computers \& Mathematics with Applications, vol. 60, no. 6, pp. 1557-1570, 2010.

[27] D. F. Li, J. X. Nan, and M. J. Zhang, "A ranking method of triangular intuitionistic fuzzy numbers and application to decision making," International Journal of Computational Intelligence Systems, vol. 3, no. 5, pp. 522-530, 2010.

[28] V. L. G. Nayagam, S. Jeevaraj, and P. Dhanasekaran, "An improved ranking method for comparing trapezoidal intuitionistic fuzzy numbers and its applications to multicriteria decision making," Neural Computing and Applications, vol. 30, no. 2, pp. 671-682, 2018.

[29] S.-P. Wan, "Multi-attribute decision making method based on possibility variance coefficient of triangular intuitionistic fuzzy numbers," International Journal of Uncertainty, Fuzziness and Knowledge-Based Systems, vol. 21, no. 2, pp. 223243, 2013.

[30] S. Das and D. Guha, "Ranking of intuitionistic fuzzy number by centroid point," Journal of Industrial and Intelligent Information, vol. 1, no. 2, pp. 107-110, 2013.

[31] X. Li and X. Chen, "Multi-criteria group decision making based on trapezoidal intuitionistic fuzzy information," $A p$ plied Soft Computing, vol. 30, pp. 454-461, 2015.

[32] V. Lakshmana Gomathi Nayagam, S. Jeevaraj, and P. Dhanasekaran, "A linear ordering on the class of trapezoidal intuitionistic fuzzy numbers," Expert Systems with Applications, vol. 60, pp. 269-279, 2016.

[33] V. L. G. Nayagam, S. Jeevaraj, and S. Geetha, "Total ordering for intuitionistic fuzzy numbers," Complexity, vol. 21, no. 2, pp. 54-66, 2016. 
[34] S. Aggarwal and C. Gupta, "Solving intuitionistic fuzzy solid transportation problem via new ranking method based on signed distance," International Journal of Uncertainty, Fuzziness and Knowledge-Based Systems, vol. 24, no. 4, pp. 483-501, 2016.

[35] K. A. Prakash, M. Suresh, and S. Vengataasalam, "A new approach for ranking of intuitionistic fuzzy numbers using a centroid concept," Mathematical Sciences, vol. 10, no. 4, pp. 177-184, 2016.

[36] V. Singh and S. P. Yadav, "Development and optimization of unrestricted LR-type intuitionistic fuzzy mathematical programming problems," Expert Systems with Applications, vol. 80, pp. 147-161, 2017.

[37] B. P. Canedo and E. R. C. Morales, "On LR-type fully intuitionistic fuzzy linear programming with inequality constraints: solutions with unique optimal values," Expert Systems with Applications, vol. 128, pp. 246-255, 2019.

[38] M. Darehmiraki, "A novel parametric ranking method for intuitionistic fuzzy numbers," Iranian Journal of Fuzzy Systems, vol. 16, no. 1, pp. 129-143, 2019.

[39] R. A. Shureshjani and M. Darehmiraki, "A new parametric method for ranking fuzzy numbers," Indagationes Mathematicae, vol. 24, no. 3, pp. 518-529, 2013.

[40] M. Xu, S. Liu, Z. Xu, and W. Zhou, "DEA evaluation method based on interval intuitionistic bayesian network and its application in enterprise logistics," IEEE Access, vol. 7, pp. 98277-98289, 2019.

[41] S. H. Razavi Hajiagha, H. Akrami, E. K. Zavadskas, and S. S. Hashemi, "An intuitionistic fuzzy data envelopment analysis for efficiency evaluation under uncertainty: case of a finance and credit institution," E a M: Ekonomie a Management, vol. 161, pp. 128-137, 2013.

[42] J. Puri and S. P. Yadav, "Intuitionistic fuzzy data envelopment analysis: an application to the banking sector in India," Expert Systems with Applications, vol. 42, no. 11, pp. 4982-4998, 2015.

[43] S. Singh, "Intuitionistic fuzzy DEA/AR and its application to flexible manufacturing systems," RAIRO-Operations Research, vol. 52, no. 1, pp. 241-257, 2018.

[44] A. Arya and S. P. Yadav, "Development of intuitionistic fuzzy super-efficiency slack based measure with an application to health sector," Computers \& Industrial Engineering, vol. 115, pp. 368-380, 2018.

[45] A. Arya and S. P. Yadav, "Development of intuitionistic fuzzy data envelopment analysis models and intuitionistic fuzzy input-output targets," Soft Computing, vol. 23, no. 18, pp. 8975-8993, 2019.

[46] A. Arya and S. P. Yadav, "A new approach to rank the decision making units in presence of infeasibility in intuitionistic fuzzy environment," Iranian Journal of Fuzzy Systems, vol. 17, no. 2, pp. 183-199, 2020.

[47] Z. Ameri, S. S. Sana, and R. Sheikh, "Self-assessment of parallel network systems with intuitionistic fuzzy data: a case study," Soft Computing, vol. 23, no. 23, pp. 12821-12832, 2019.

[48] A. Charnes, W. W. Cooper, and E. Rhodes, "Measuring the efficiency of decision making units," European Journal of Operational Research, vol. 2, no. 6, pp. 429-444, 1978.

[49] A. Emrouznejad, M. Tavana, and A. Hatami-Marbini, "The state of the art in fuzzy data envelopment analysis," in Performance Measurement with Fuzzy Data Envelopment Analysis, vol. 309, pp. 1-45, Springer, Berlin, Germany, 2014.

[50] C. B. Chen and C. M. Klein, "A simple approach to ranking a group of aggregated fuzzy utilities," IEEE Transactions on
Systems, Man and Cybernetics, Part B (Cybernetics), vol. 27, no. 1, pp. 26-35, 1997.

[51] X. Wang and E. E. Kerre, "Reasonable properties for the ordering of fuzzy quantities (I)," Fuzzy Sets and Systems, vol. 118, no. 3, pp. 375-385, 2001a.

[52] M. Ma, M. Friedman, and A. Kandel, "A new fuzzy arithmetic,” Fuzzy Sets and Systems, vol. 108, no. 1, pp. 83-90, 1999.

[53] A. A. Foroughi and R. A. Shureshjani, "Solving generalized fuzzy data envelopment analysis model: a parametric approach," Central European Journal of Operations Research, vol. 25, no. 4, pp. 889-905, 2017.

[54] W. D. Cook and J. Zhu, Data Envelopment Analysis-A Handbook on the Modeling of Internal Structures and Networks, Springer, New York City, NY, USA, 2014.

[55] C. Kao and S.-N. Hwang, "Efficiency decomposition in twostage data envelopment analysis: an application to non-life insurance companies in Taiwan," European Journal of Operational Research, vol. 185, no. 1, pp. 418-429, 2008.

[56] C. Kao, "Efficiency decomposition for parallel production systems," Journal of the Operational Research Society, vol. 63, no. 1, pp. 64-71, 2012. 